\title{
How dusty was the last glacial maximum over Europe?
}

\author{
Denis-Didier Rousseau a, b, *, Pierre Antoine ${ }^{c}$, Youbin Sun ${ }^{\mathrm{d}}$ \\ a Laboratoire de Météorologie Dynamique (CNRS and Institute Pierre Simon Laplace, IPSL), Ecole Normale Supérieure, Paris Sciences \& Lettres (PSL) \\ Research University, 75005, Paris, France \\ ${ }^{\mathrm{b}}$ Lamont-Doherty Earth Observatory of Columbia University, Palisades, NY, 10964, USA \\ ${ }^{c}$ Laboratoire de Géographie Physique, Environnements quaternaires et actuels, CNRS, 1 place A. Briand, 92195, Meudon Cedex, France \\ d State Key Laboratory of Loess and Quaternary Geology, Institute of Earth Environment, Chinese Academy of Science, Xi'an, 710061, China
}

\section{A R T I C L E I N F O}

\section{Article history:}

Received 4 August 2020

Received in revised form

9 December 2020

Accepted 15 December 2020

Available online 23 January 2021

Handling Editor: Professor Xiaoping Yang

\section{Keywords:}

Loess

Last glacial maximum

Eurasia

Sedimentation and mass accumulation rates

Millennial scale variability

\begin{abstract}
A B S T R A C T
Our study focuses on European loess sequences, particularly the eolian intervals in between the observed pedogenic units. The classical concept of soil formation from parent material is reformulated to estimate of the duration and the associated sedimentation rate (SR) and mass accumulation rate (MAR) of these paleodust intervals. We show that the Greenland Stadial (GS) duration in European loess deposits includes the thickness of the overlying pedogenic unit, which in fact developed downward into the upper part of the eolian unit. The lower stratigraphical limit of the eolian unit overlying the pedogenic unit corresponds to the restart of the dust sedimentation of the younger GS. We illustrate this interpretation first by computing both SRs and MARs first for the Nussloch key sequence, the most complete European series. The correlation between Nussloch and other European loess sequences, located along a $1800 \mathrm{~km}$ longitudinal transect, allows computation of SR and MAR for several identified GS events. Comparing GS from marine and ice core records, our study shows that the two last Bond cycles are preserved in every European eolian record.

Bulk SR and MAR are estimated and compared for these two Bond cycles, showing the highest SRs and MARs in western Europe. These indices also indicate that the last stadials, embedding an Henrich event, were not the dustiest in every Bond cycle. Our estimated MAR also differ from previously published computations, which did not take into account the various pedogenic units present in the studied loess sequences. The bulk SR and MAR estimates computed for the two last Bond Cycles from Chinese sequences from the Loess Plateau indicate lower atmospheric dust than in Europe during the Last Glacial Maximum. SR and MAR estimates computed from the fine-grained material for European records fit with Earth System model reconstructions.
\end{abstract}

() 2020 Elsevier Ltd. All rights reserved.

\section{Introduction}

Abrupt climate changes during the last glaciation are expressed as rapid transitions between Greenland stadials (cold stages - GSs) and Greenland interstadials (warm stages - GIs), characterized by large amplitude warming during GIs (Kindler et al., 2014). These abrupt changes were originally marked by iceberg discharges in the North Atlantic named Heinrich events (HE), which impacted the climate and the ocean circulations of the Northern Hemisphere. Some of these massive discharges reached southward to the Iberian

\footnotetext{
* Corresponding author. Laboratoire de Météorologie Dynamique (CNRS and Institute Pierre Simon Laplace, IPSL), Ecole Normale Supérieure, Paris Sciences \& Lettres (PSL) Research University, 75005, Paris, France.

E-mail address: denis.didier.rousseau@Imd.ipsl.fr (D.-D. Rousseau).
}

margin with significant cooling the sea surface temperature and reducing salinity (Bard et al., 2000). By correlating the climate records from the Greenland ice-cores with North Atlantic sediments, Bond et al. (1993) identified a particular succession of the Dansgaard-Oeschger cycles (DO), associated with observed GIs and GS. The long-term cooling cycles were later named Bond Cycles by Broecker (1994), which are characterized by an asymmetrical and sawtooth-like pattern, similar in shape to an individual DO cycle with a duration of about 10-15 ka. These long-term cooling cycles start with a long and strong GI and end with the coldest GS embedding an HE. The general cooling trend of these long-term cycles is associated with the regrowth of the Laurentide ice-sheet after every HE (Alley, 1998; Alley et al., 1999; Clark et al., 2007).

The climate in Europe has been strongly influenced by millennial climate changes related to variations in the Atlantic Meridional 
Overturning Circulation (AMOC), the extent of northern ice shelves and sea-ice, which also affected the moisture sources of precipitation on the Greenland ice sheet, and therefore its growth (Boers et al., 2018). These variations in the extent of the sea ice during the last climatic cycle (LCC, about 130-15 kyr) affected the westerlies and the position of the polar jet stream, and consequently storm track trajectories (Lohmann et al., 2020). The resulting continental records have been mainly interpreted in terms of temperature, precipitation or vegetation changes related to the variation between GIs and GSs (Sanchez-Goni et al., 2008). The $\delta^{18} \mathrm{O}$ increases in the Greenland NGRIP ice core record, named Dansgaard-Oeschger events (DOE), correspond to an average temperature difference of about $+11.8{ }^{\circ} \mathrm{C}$ on the top of the Greenland ice sheet (Kindler et al., 2014), associated with substantial reorganizations of the ecosystems in mid-latitude Europe (Harrison and Sanchez Goñi, 2010; Sanchez Goñi and Harrison, 2010; Sanchez Goñi et al., 2017). Furthermore, the presence of ice sheets and ice caps over Great Britain, Scandinavia and the Alps enhanced the zonal circulation during GS, as supported by numerical experiments (Sima et al., 2009, 2013).

Loess sequences, eolian deposits, are well developed all over Europe, especially between $48^{\circ}$ and $52^{\circ} \mathrm{N}$, where one or more climate cycles have been recorded (Kukla, 1970) (Fig. 1). During the LCC intensive deposition of dust over Europe was accompanied by a reduction or practically absence of arboreal cover in NW Europe during GSs (Woillard, 1978), and by sea-level lowering exposing large areas of the continental shelf to eolian erosion. Strong increases in fluvial transport and sedimentation by periglacial braided rivers released large amounts of material available for deflation (Lautridou, 1985; Rousseau et al., 2018; Vandenberghe and Maddy, 2001). Geochemical study of LGM samples from European loess sequences located along a longitudinal transect from Brittany to Ukraine shows that these deposits correspond only to a regional (hundreds of kilometers) transport of the available eolian material (Rousseau et al., 2014). Extensive multidisciplinary investigations of European loess deposits along a longitudinal transect at $50^{\circ} \mathrm{N}$ (Fig. 1) reveal that the millennial-scale climate variations observed in the North-Atlantic marine and Greenland ice-core records of the LCC are well preserved in the succession of paleosol-loess alternations or doublets (Rousseau et al., 2002, 2007). Loess units represent GSs, while GIs correspond to either paleosols of various types or slightly pedogenic horizons, depending on the GI duration (Rousseau et al., 2017a, 2017b). Loess deposits are interpreted to correspond to coarse paleodust transported at rather low elevations in the atmospheric boundary layer (from about 300 to a maximum $3000 \mathrm{~m}$ ) at local to regional scales (Rousseau et al., 2014). Finer paleodust may have been transported at much higher elevation by the general circulation, allowing deposition at higher latitudes (Antoine et al., 2009b; Pye, 1995; Pye and Zhou, 1989; Rousseau et al., 2014, 2017b; Ujvari et al., 2015; Vandenberghe, 2013). Along the $50^{\circ} \mathrm{N}$ transect, the Nussloch profile yields the most-detailed record of the paleosol-loess alternation of the LCC making this record a key European sequence (Antoine et al., 2001, 2009b; Moine et al., 2017; Rousseau et al., 2002, 2007, 2017a, 2017b). The succession of paleosol-loess doublets in Nussloch is characterized by a variable thickness and the different types of paleosols in the sequences, ranging from western Europe eastward to Ukraine (Rousseau et al., 2011). These spatial characteristics help to evaluate the duration of the paleosol formation, and develop a more precise chronology of the eolian deposition over the vast European territory. Although a fundamental element in the definition of loess stratigraphy, is to identify pedogenic units, they are very limited in the construction of reliable timescales for further model-data comparisons (Rousseau et al., 2017a, 2017b).

In this study, we propose new estimates of the sedimentation rate (SR) and mass accumulation rate (MAR) of European loess sequences. First, we reconstruct these parameters for the reference sequence of Nussloch, especially for the various identified GS equivalents during which dust deposition occurred. Next, we expand this study to other European records based on their stratigraphical correlations with Nussloch through observed marker layers and available dates. Finally, our estimates are compared with previous published estimates for other European loess series, and with Chinese loess sequences. A comparison is also made with dust deposition reconstructions in Europe from the Last Glacial Maximum (LGM) modeling studies (Kageyama et al., 2018).

\section{The Nussloch loess sequence}

The Nussloch loess sequence is the key reference for the European LCC (Zöller and Löscher, 1997). It is located on the right bank of
0

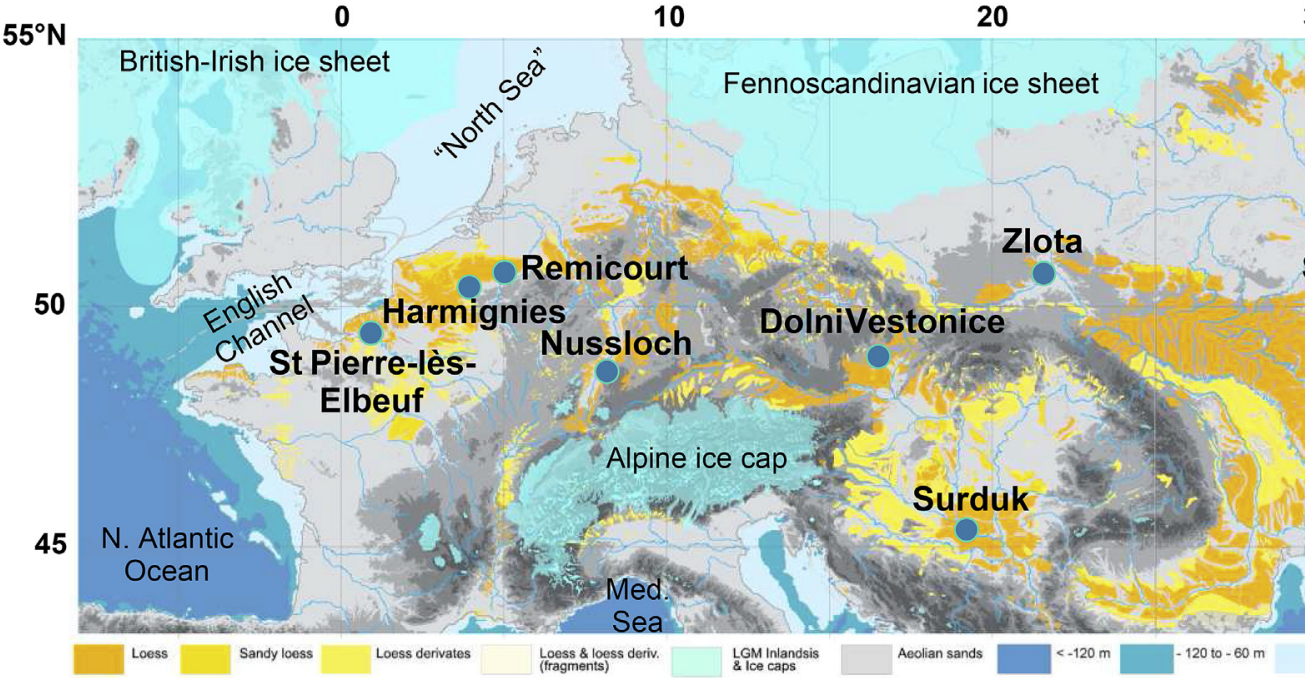

30

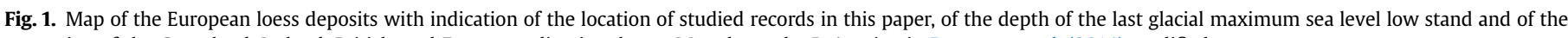
expansion of the Greenland, Iceland, British, and Fennoscandian ice sheets. Map drawn by P. Antoine in Rousseau et al. (2014) modified. 
the Rhine River, south of Heidelberg in Germany, on a plateau overhanging a wide floodplain (Fig. 1). The loess deposits are distributed along ridges oriented NW-SE (Antoine et al., 2001) in which a succession of alternating paleosol-loess doublets have been identified (Antoine et al., 2001, 2009b; Bibus et al., 2007; Rousseau et al., 2002, 2007, 2017a, 2017b). These doublets are correlated very precisely with the succession of GI-GS doublets from the Greenland NGRIP ice core through ${ }^{14} \mathrm{C}$ dates on earthworm granules (Moine et al., 2017) supporting previous stratigraphical assignment of the pedogenic units, mostly for the highly dilated sedimentary section covering the $40 \mathrm{ka}$ to $18 \mathrm{ka}$ interval. Erosion is not evident between the pedogenic and the loess units from the sampling profile (see Supplementary figure 2 in (Rousseau et al., 2017a)) except for the occurrence of a large thermokarst erosion gully at the base of the Middle Pleniglacial record (TK2 in (Antoine et al., 2009a)). Timing of paleosol formation is in agreement with the detailed and well-dated records of GI in Turkish cave deposits located at about the same longitude as our easternmost studied loess sequence (Stayky in Ukraine (Rousseau et al., 2011)). In these speleothems, GIs are expressed by the responses of local ecosystems by the expansion of C3 plants and higher soil activity (Fleitmann et al., 2009). Interestingly, the identified pedogenic units in Nussloch range between brown boreal paleosols to tundra gleys to embryonic oxidized horizons. They are characterized by the lowest $\delta^{13} \mathrm{C}$ from the preserved organic matter in the sediment (Hatté et al., 1998), corresponding to GI 17 to GI 2, in addition to the identified interglacial $\mathrm{Bt}$ horizon at the base of the sequence (Antoine et al., 2009b) (Fig. 2).

The identification of different types of paleosols and pedogenic horizons in Nussloch, is fundamental. We have used the established correlation to the Greenland records (Moine et al., 2017; Rousseau et al., 2017a, 2017b) to estimate the maximum amount of time needed to reach their different degrees of maturation and development. To identify these time lapses more precisely, we employed two independent NGRIP $\delta^{18} \mathrm{O}$ and dust concentration data to indicate variations of atmospheric temperature and dustiness in the Greenland area, respectively. Our correlating strategy slightly differs from the definition of a GI event duration applied in other studies, where the sharp end of the $\delta^{18} \mathrm{O}$ decrease alone defines the end of a GI. Our correlation was conducted both visually and algorithmically to both proxy records (i.e., the GIs are defined to last from the beginning of the abrupt $\delta^{18} \mathrm{O}$ increase or the dust concentration decrease until the time when $\delta^{18} \mathrm{O}$ or dust return to their initial value before the GI onset).

Rousseau et al. (2017a) have analyzed the GIs in both $\delta^{18} \mathrm{O}$ and dust NGRIP records, showing that the abrupt change in these proxies averages from 50 to 150 years. The proxies have two different origins with $\delta^{18} \mathrm{O}$ mostly from the North Atlantic surface water and the dust predominantly from the Chinese northern deserts. From a statistical point of view the lead or lag between the start of the change of these two proxies is negligible (2017a). Therefore, we assume that although a short lag of several decades could exist between the changes in the $\delta^{18} \mathrm{O}$ in Greenland ice core and the start of the paleosol or tundra gley formation, such a lag cannot be distinguished in the centennial-resolved loess deposits. Similarly, the GIs/GS transitions in the $\delta^{18} \mathrm{O}$ and the dust records, are rather contemporaneous (2017a). Therefore the paleosol and tundra gley formation ended at the same time, i.e., as indicated in the Rasmussen et al. (2014) chronology. To reduce the uncertainty about this time interval assignment, extremely high-resolution sampling of every paleosol and tundra gley could be performed but age errors between these two records still cannot be well resolved due to their different sensitivity to climate changes. Consequently, we reliably estimate the duration of the GIs as the maximum time for the development of the paleosols observed in Nussloch, (Rousseau et al., 2017a).

Subsequently, Rousseau et al. (2017b), proposed an alternative way of investigating the Nussloch eolian deposits in order to convert it into terms of a deposition budget. As expected, when taking into account paleosol or pedogenic unit formation, SR and MAR cannot be estimated by assuming a continuous accumulation of eolian deposits between two available dates as is classically performed for marine, ice or lake records, Following the detailed correlations defined between Nussloch and NGRIP stratigraphies (Fig. 2), with one pedogenic unit corresponding to a particular GI, (Moine et al., 2017; Rousseau et al., 2002, 2007, 2017a, 2017b), we applied to the loess sequence the dates obtained for every start and end of a GI, as determined by Rasmussen et al. (2014). This represents a reliable compromise with our estimates described previously, following the acknowledged synchronicity of DO events in numerous worldwide paleoclimate records (Adolphi et al., 2018). We considered that LCC paleosols in European loess sequences at $50^{\circ} \mathrm{N}$, were developed from the underlying loess deposits after the eolian sedimentation ceased, and therefore should be considered as the upper part of the eolian deposits (Taylor and Lagroix, 2015; Taylor et al., 2014; Vandenberghe et al., 2014). Moreover, the eolian sequences on top of the developed paleosols can be considered to have restarted after the formation of each pedogenic units. This makes the time evolution of these loess sequences nonlinear and a bit more complex than the classical continuous sedimentation. Therefore, considering that the stratigraphical succession of the identified units was developed on the underlying parent material, the time's arrow, from the bottom to the top of the sequence, always changed direction during the pedogenic development by moving downward in the sequence, and finally moved upward from the top of the paleosols. Such conceptual evolution of the time's arrow completely differs from that happens in Asian loess sequences, and other paleoclimatological records, i.e., lake, marine or ice cores where time only goes upward (Kukla and Koci, 1972; Rousseau et al., 2017a). For that reason, a determined eolian interval, equivalent to a GS, corresponds to both the thickness of the visible loess layer and the thickness of the layer, which underwent soil forming processes (blue arrow in Fig. 2). The pedogenesis itself fits with the GI duration (red arrow in Fig. 2). As a result, the Nussloch stratigraphy can be read as expressed in Table 1, allowing an improved estimate the SRs and the MARs that are required for comparison with other loess records and also with model outputs.

In the 60-23 ka b2k interval, corresponding to four long-term cooling cycles recognized by, Bond et al. (1993) we have labeled "Bond Cycle a" (BCA) the cycle lasting from GI4 to GS3/HE2, "Bond Cycle b" (BCB) the cycle lasting from GI8 to GS5/HE3, "Bond cycle c" (BCC) the cycle lasting from GI12 to GS9/HE4, and "Bond Cycle d" (BCD) the cycle lasting from GI14 to GS13/HE5 in the Nussloch loess sequence (Fig. 2). By reference to Rasmussen et al. (2014) chronology, SR varies in the whole Nussloch sequence between 186 and $22 \mathrm{~cm} / \mathrm{kyr}$ (Fig. 2, Table 1). More precisely, according to the correlation of the Nussloch loess sequence with NGRIP ice-core record, SR varies between 186 and $111 \mathrm{~cm} / \mathrm{kyr}$ during BCA, lasting between $28,900 \mathrm{yr}$ and 23,340 yr b2k, 127-46 cm/kyr during BCB, lasting between $38,220 \mathrm{yr}$ and 28,900 yr b2k, 165-22 cm/kyr during BCC, lasting between $46,860 \mathrm{yr}$ and 38,220 yr b2k, and 224-59 cm/kyr during BCD, lasting between $54,220 \mathrm{yr}$ and $46,860 \mathrm{yr}$ b2k. Interestingly, the maximum SR value in every Bond Cycle is not reached at its end, when HE was released in the North Atlantic, but at its penultimate stadial (Table 1). This appears in agreement with reconstructions of wind speed and environmental conditions which show coldest and windest GSs compared to HEs (Sima et al, 2009, 2013). Such results also agree with the interpretation of the Black Sea temperature response to glacial millennial climate variability 


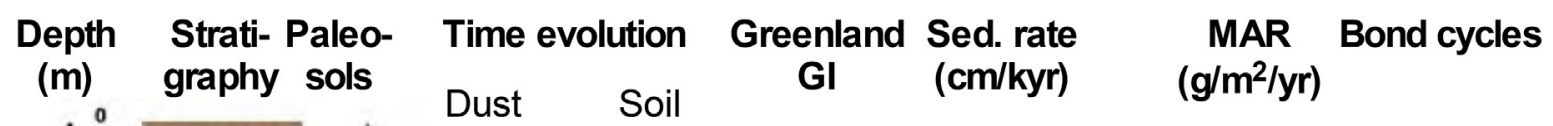
(m) graphy sols
Dust Soil
GI (cm/kyr)
(g/m²/yr)

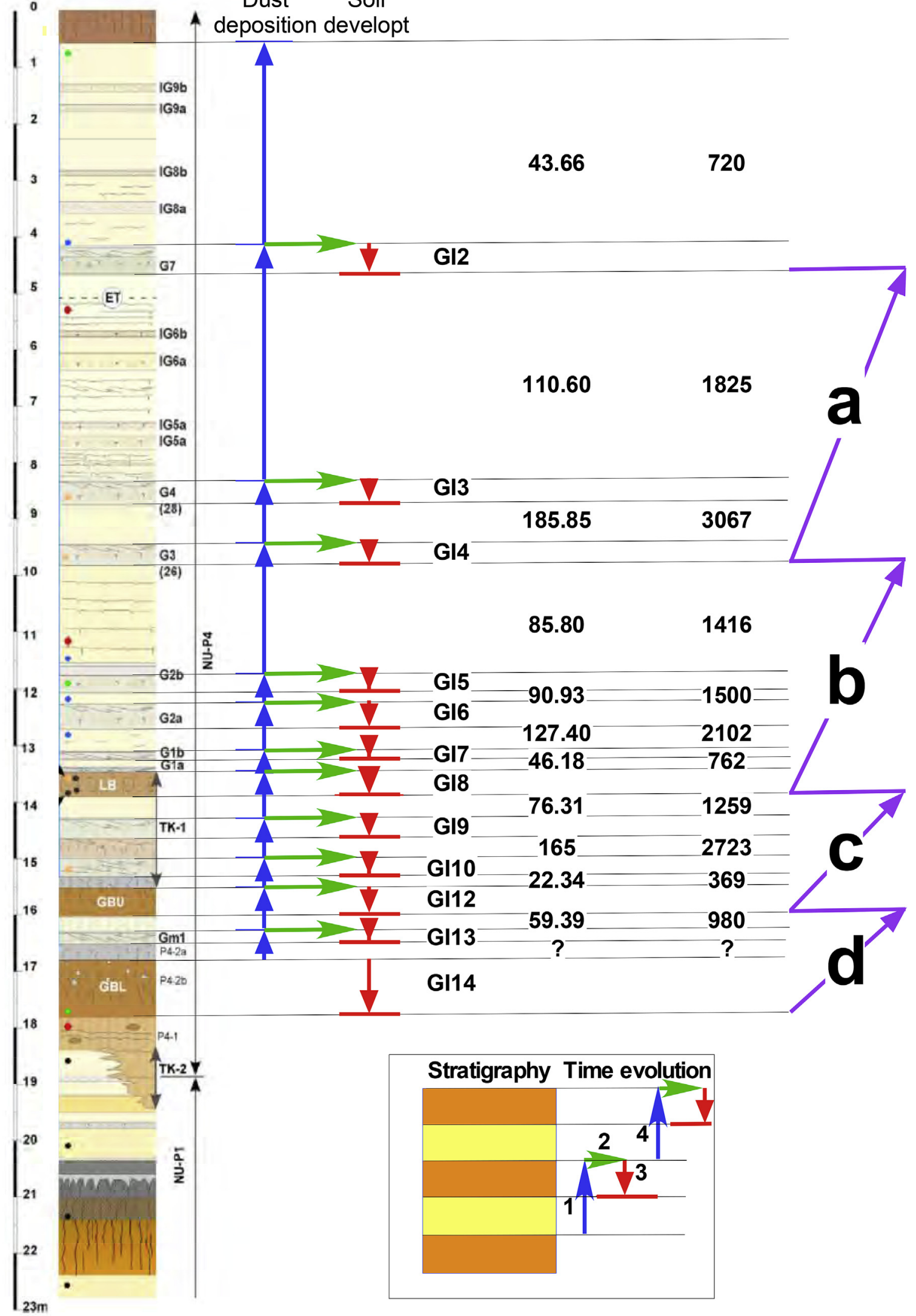


during the LCC, indicating that HE-stadials in this area were cooler than the regular stadials (Wegwerth et al., 2015), as also indicated by Ganopolski and Rahmstorf (2001) and Zhang et al. (2014) modeling experiments.

To convert our SRs into MARs, we applied the equation defined by Kohfeld and Harrison (2003), MAR = f*SR*BD with $\mathrm{SR}=$ sedimentation rate, $\mathrm{f}=$ fraction of sediment which is eolian, and $\mathrm{BD}=$ the bulk density considered as constant with a value of $1.65 \mathrm{~g} / \mathrm{cm}^{3}$ for loess (Pye, 1987). Frechen et al. (2003) previously applied this equation to European loess series, among which are some of the sequences we analyzed in the present study for further comparison. Therefore, the bulk MAR in Nussloch, with $f=1$, because considering bulk samples, varies between 2578 and $414 \mathrm{~g} /$ $\mathrm{m}^{2} / \mathrm{yr}$ (Fig. 2; Table 1) over the 60-23 ka b2k interval. Similarly to our SR computation, the bulk MAR can be decomposed into 3067-1825 g/m²/yr during BCA, 2102-762 g/ $\mathrm{m}^{2} / \mathrm{yr}$ during BCB, 2723-369 g/ $\mathrm{m}^{2} / \mathrm{yr}$ during BCC, and 3702-980 g/ $\mathrm{m}^{2} / \mathrm{yr}$ during BCD. These estimates are by far different than those from Frechen et al. (2003), i.e. $6129-1213 \mathrm{~g} / \mathrm{m}^{2} / \mathrm{yr}$ also for Nussloch. These latter values were, however calculated using a simple accumulation age model without including the soil developments on top of the lower eolian units as applied in our study. Notably, both our new maximum and minimum estimates indicate much lower value by a factor of 2-3 respectively, differences that are not negligible.

From our study, BCA is the time interval showing the highest values in both bulk SR and MAR in Nussloch. This seems to be intuitively evident because this time interval corresponds to the maximum dust concentration in Greenland (Rasmussen et al., 2014). This period was indeed apparently the most favorable for dust emission, transport and deposition in the Northern Hemisphere because of the coldest and driest conditions recorded (Clark et al., 2009) and the largest deflation areas available in relation to the lowest sea level (Grant et al., 2012). BCB also shows high values, but has a much smaller magnitude than that obtained for BCA. However, as this result is only evident in Nussloch, testing with other high-resolution European loess sequences is needed.

\section{European loess sequences}

Some other high-resolution loess sequences located at $50^{\circ} \mathrm{N}$ were investigated at high resolution to detect the occurrence of paleosols, markers of millennial scale variations during the LCC (Rousseau et al., 2018). Distributed in the same latitude from the North of France to Ukraine, several representative loess records are selected for comparison with the Nussloch key sequence, such as St. Pierre-lès-Elbeuf and other related sequences (France) (Antoine et al., 1999, 2014, 2016), Harmignies and Remicourt (Belgium) (Antoine et al., 2001), Dolni Vestonice (Czech Republic) (Antoine et al., 2013), Zlota (Poland) (Moska et al, 2015, 2018) and Stayky (Ukraine) (Rousseau et al., 2011). We included the Serbian sequence Surduk located in the Carpathian Basin for comparison because this region should have experienced a particular circulation dynamics during the LCC linked to its particular geographical configuration (Antoine et al., 2009a). The basin is almost closed due the presence of the Carpathian arch to the north and the east, the Alpine Ice Cap covers all the Alps to the west and the Dinarides Alps to the south.
Sima et al., 2009, 2013 have demonstrated a similar pattern in dust emission and transport all over Europe during GS and GI conditions showing that with the vegetation development in late Spring, the emission of dust was prevented, and therefore deposited at further distance. A comparison of the modeling results and the geochemical study of sequences along a longitudinal transect from Brittany towards Ukraine, Rousseau et al. (2014) demonstrated that the eolian deposits were mainly originated from various regional sources along this longitudinal transect including the English Channel. While dust pulses may have occurred very locally, the general pattern remains comparable as supported by the numerous dates obtained in the studied sequences.

The correlation of the LCC record among different sequences relies upon the identification of key paleosols horizons, based on the published ${ }^{14} \mathrm{C}$ and luminescence dates and the grain size variations of these loess records. Preliminary correlations have been discussed in the previous papers presenting the studied sites (Antoine et al., 1999, 2001, 2009b, 2013, 2016; Moska et al., 2015, 2018; Rousseau et al., 2011). The resulting correlations between Nussloch and the other sequences has been presented in Rousseau et al. (2017a), see description in the manuscript with references herein and the supplementary figure S1 illustrating the correlations between the identified groups of horizons). The thickness of the identified units and their time assignment according to NGRIP chronology are presented in Tables 1 and 2. By assigning the NGRIP dates to the various units of the Nussloch sequence after considering identified site-specificity, we infer that $\mathrm{BCA}$ and $\mathrm{BCB}$, the dustiest intervals of the LCC in Nussloch, are preferably preserved as well in all the sequences, with the exception of Dolni Vestonice where only BCA is best recorded and useable for comparisons. We investigate the variations of both SR and MAR along this longitudinal transect for the time intervals that these two Bond cycles represent (Figs. 3 and 4, S1; Table 2).

\subsection{Sedimentation rates (SRs) (Fig. 3)}

Along the $50^{\circ} \mathrm{N}$ and if the values obtained at the site of St Pierrelès-Elbeuf, which is a composite record, and very different from the other sites, SR ranges between 231 and $104 \mathrm{~cm} / \mathrm{kyr}$ for the maximum, and between 57 and $14 \mathrm{~cm} / \mathrm{kyr}$ for the lowest values. When considering the two most recent Bond Cycles, BCA always shows the highest values either for the maximum and the minimum ranges (231-104 and 137-51 cm/kyr) than BCB (204-84 and $57-20 \mathrm{~cm} / \mathrm{kyr}$ ) (Table 2, Fig. 3). Although the general circulation was rather controlled by the westerlies, a gradient emerges from the data when considering the bulk sediment with higher SRs westward compared to central and eastern Europe (Fig. 3). No specific pattern seems to be associated to Surduk, either during BCA and $\mathrm{BCB}$.

\subsection{Mass accumulation rates (MARs) (Fig. 4)}

Along the studied transect, applying the same formula as for Nussloch and keeping St Pierre-lès-Elbeuf aside, MAR ranges between 3803 and $1710 \mathrm{~g} / \mathrm{m}^{2} / \mathrm{yr}$ for the maximum and between 945 and $325 \mathrm{~g} / \mathrm{m}^{2} / \mathrm{yr}$ for the minimums. Considering the Bond cycles,

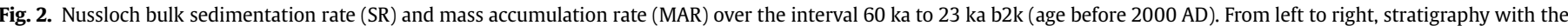

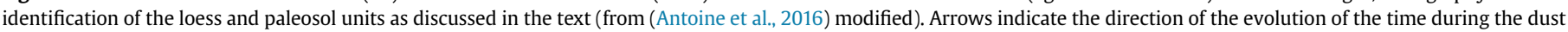

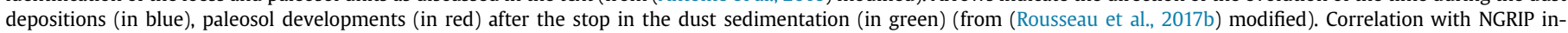

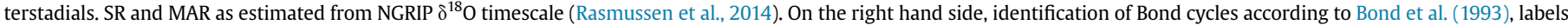

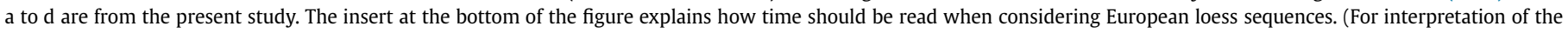
references to colour in this figure legend, the reader is referred to the Web version of this article.) 
Table 1

Nussloch data. From left to right, Stratigraphy expressed in terms of thickness of the eolian deposits, including the paleosol development from the top of the loess units. NGRIP correspondence, Nussloch individual GS bulk SR and MAR estimates for the four Bond cycles comprised between 60 and 15ka b2k (Rasmussen et al., 2014).

\begin{tabular}{|c|c|c|c|c|c|c|c|c|c|c|c|c|c|}
\hline Eolian sedimentation & Paleosols & $\begin{array}{l}\text { Thickness } \\
\text { depth }(\mathrm{m})\end{array}$ & $\begin{array}{c}\text { Thickness } \\
\text { for } \\
\text { MAR/SR }\end{array}$ & $\begin{array}{c}\text { Age end } \\
\text { Rasmussen (yr) }\end{array}$ & $\begin{array}{l}\text { Age start } \\
\text { Rasmussen } \\
\text { (yr) }\end{array}$ & $\begin{array}{c}\text { Duration } \\
\text { Rasmussen } \\
\text { (yr) }\end{array}$ & $\begin{array}{l}\text { Sedimentation rate } \\
\text { Rasmussen } \\
(\mathrm{mm} / \mathrm{yr})\end{array}$ & $\begin{array}{l}\text { Age end } \\
\text { dust (yr) }\end{array}$ & $\begin{array}{l}\text { MAR Rasmussen } \\
(\mathrm{g} / \mathrm{m} 2 / \mathrm{yr})\end{array}$ & $\begin{array}{l}\text { MAR dust } \\
(\mathrm{g} / \mathrm{m} 2 / \mathrm{yr})\end{array}$ & $\begin{array}{l}\text { SR Rasmussen } \\
\mathrm{cm} / \mathrm{kyr}\end{array}$ & $\begin{array}{c}\text { sed rate } \\
\text { dust } \mathrm{cm} / \mathrm{kyr}\end{array}$ & \\
\hline \multirow[t]{2}{*}{ Top sequece - Top G7 (GI2) } & & 3.589 & 3.589 & 15,000 & 23,220 & 8220 & 0.44 & 15,000 & 720 & 723 & 43.66 & 43.82 & Bond cycle 0 \\
\hline & G7 (GI2) & 0.508 & & 23,220 & 23,340 & 120 & & 23,190 & & & & & \\
\hline \multirow[t]{2}{*}{ Top G7 - Top G4 (GI3) } & & 4137 & 4.645 & 23,340 & 27,540 & 4200 & 1.11 & 23,370 & 1825 & 1851 & 110.60 & 112.20 & Bond cycle a \\
\hline & G4 (GI3) & 0.411 & & 27,540 & 27,780 & 240 & & 27,510 & & & & & \\
\hline \multirow[t]{2}{*}{ Top G4 - Top G3 (GI4) } & & 1.113 & 1.524 & 27,780 & 28,600 & 820 & 1.86 & 27,800 & 3067 & 3445 & 185.85 & 208.77 & \\
\hline & G3 (GI4) & 0.387 & & 28,600 & 28,900 & 300 & & 28,530 & & & & & \\
\hline \multirow[t]{2}{*}{ Top G3 - Top G2b (GI5) } & & 2.306 & 2.694 & 28,900 & 32,040 & 3140 & 0.86 & 28,910 & 1416 & 1507 & 85.80 & 91.32 & Bond cycle b \\
\hline & G2b (GI5) & 0.290 & & 32,040 & 32,500 & 460 & & 31,860 & & & & & \\
\hline \multirow[t]{2}{*}{ Top G2b - Top G2a (GI6) } & & 0.492 & 0.782 & 32,500 & 33,360 & 860 & 0.91 & 32,500 & 1500 & 1593 & 90.93 & 96.54 & \\
\hline & G2a (GI6) & 0.444 & & 33,360 & 33,740 & 380 & & 33,310 & & & & & \\
\hline \multirow[t]{2}{*}{ Top G2a - Top G1b (GI7) } & & 0.831 & 1.274 & 33,740 & 34,740 & 1000 & 1.27 & 33,750 & 2102 & 2416 & 127.40 & 146.44 & \\
\hline & G1b (G7) & 0.145 & & 34,740 & 35,480 & 740 & & 34,620 & & & & & \\
\hline \multirow[t]{2}{*}{ Top G1b - Top LB (GI8) } & & 0.363 & 0.508 & 35,480 & 36,580 & 1100 & 0.46 & 35,490 & 762 & 798 & 46.18 & 48.38 & \\
\hline & LB (GI8) & 0.452 & & 36,580 & 38,220 & 1640 & & 36,540 & & & & & \\
\hline \multirow[t]{2}{*}{ Top LB - Top Gm3 (GI9) } & & 0.831 & 1.282 & 38,220 & 39,900 & 1680 & 0.76 & 38,220 & 1259 & 1259 & 76.31 & 76.31 & Bond cycle c \\
\hline & Gm3 (GI9) & 0.347 & & 39,900 & 40,160 & 260 & & 39,900 & & & & & \\
\hline \multirow[t]{2}{*}{ Top Gm3 - Top Gm2 (GI10) } & & 0.710 & 1.056 & 40,160 & 40,800 & 640 & 1.65 & 40,180 & 2723 & 2904 & 165.00 & 176.00 & \\
\hline & $\mathrm{Gm} 2(\mathrm{GI} 10)$ & 0.298 & & 40,800 & 41,460 & 660 & & 40,780 & & & & & \\
\hline \multirow[t]{2}{*}{ ? } & & & 0.298 & 41,460 & 42,240 & 780 & & 41,490 & & & & & \\
\hline & TK1 (GI11) & & & 42,240 & 43,340 & 1100 & & 42,200 & & & & & \\
\hline \multirow[t]{2}{*}{ unitX - Top GBU (GI12) } & & 0.210 & 0.210 & 43,340 & 44,280 & 940 & 0.22 & 43,360 & 369 & 417 & 22.34 & 25.30 & \\
\hline & GBU (GI12) & 0.508 & & 44,280 & 46,860 & 2580 & & 44,190 & & & & & \\
\hline \multirow[t]{2}{*}{ Top GBU - Top Gm1 (GI13) } & & 0.371 & 0.879 & 46,860 & 48,340 & 1480 & 0.59 & 46,870 & 980 & 936 & 59.39 & 56.71 & Bond cycle $\mathrm{d}$ \\
\hline & Gm1 (GI13) & 0.218 & & 48,340 & 49,280 & 940 & & 48,420 & & & & & \\
\hline \multirow[t]{2}{*}{ Top Gm1 - Top GBL (GI14) } & & 0.500 & 0.718 & 49,280 & 49,600 & 320 & 2.24 & 49,300 & 3702 & 1669 & 224.38 & 101.13 & \\
\hline & GBL (GI14) & 1.016 & & 49,600 & 54,220 & 4620 & & 50,010 & & & & & \\
\hline Mean & & & & & & 1508.46 & 1.03 & & 1702 & 1627 & 103.15 & 98.58 & \\
\hline standard dev & & & & & & 1798.86 & 0.62 & & 1024 & 915 & 62.09 & 55.44 & \\
\hline $\operatorname{Max}$ & & & & & & 8220.00 & 2.24 & & 3702 & 3445 & 224.38 & 208.77 & \\
\hline Min & & & & & & 120.00 & 0.22 & & 369 & 417 & 22.34 & 25.30 & \\
\hline
\end{tabular}


Table 2

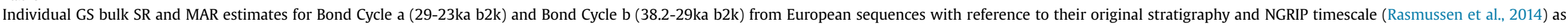
reference.

\begin{tabular}{|c|c|c|c|c|c|c|c|c|c|c|c|c|c|c|c|c|c|c|c|c|c|c|c|c|}
\hline \multicolumn{2}{|c|}{ Reference strati (Nussloch) } & \multicolumn{3}{|c|}{ NGRIP (Greenland) } & \multicolumn{4}{|c|}{ Nussloch (Germany) } & \multicolumn{5}{|c|}{ St Pierre-lès-Elbeuf (France) } & \multicolumn{5}{|c|}{ Harmignies (Belgium) } & \multicolumn{5}{|c|}{ Remicourt (Belgium) } & \multirow{2}{*}{$\begin{aligned} & \text { Bond } \\
= & \text { Cycle }\end{aligned}$} \\
\hline $\begin{array}{c}\text { Eolian } \\
\text { sedimentation }\end{array}$ & Paleosols & $\begin{array}{l}\text { Age } \\
\text { end } \\
\text { (yr) }\end{array}$ & $\begin{array}{l}\text { Age } \\
\text { start } \\
(\mathrm{yr})\end{array}$ & $\begin{array}{c}\text { Duration } \\
\text { (yr) }\end{array}$ & $\begin{array}{l}\text { Thickness } \\
\text { depth (m) }\end{array}$ & $\begin{array}{l}\text { Thickness } \\
\text { for } \\
\text { MAR/SR }\end{array}$ & $\begin{array}{c}\mathrm{SR} \\
(\mathrm{cm} / \mathrm{kyr})\end{array}$ & $\begin{array}{c}\text { MAR } \\
(\mathrm{g} / \mathrm{m} 2 / \mathrm{yr})\end{array}$ & Paleosols & $\begin{array}{l}\text { Thickness } \\
\text { depth (m) }\end{array}$ & $\begin{array}{l}\text { Thickness } \\
\text { for } \\
\text { MAR/SR }\end{array}$ & $\begin{array}{c}\mathrm{SR} \\
(\mathrm{cm} / \mathrm{kyr})\end{array}$ & $\begin{array}{c}\mathrm{MAR} \\
(\mathrm{g} / \mathrm{m} 2 / \mathrm{yr})\end{array}$ & Paleosols & $\begin{array}{l}\text { Thickness } \\
\text { depth (m) }\end{array}$ & $\begin{array}{l}\text { ess } \text { Thickne } \\
\text { m) } \\
\text { for } \\
\text { MAR/S }\end{array}$ & $\begin{array}{ll}\text { ess } & \text { SR } \\
& (\mathrm{cm} / \mathrm{ky} \\
\mathrm{SR} & \end{array}$ & $\begin{array}{c}\text { MAR } \\
\text { (yr) }(\mathrm{g} / \mathrm{m} 2 / \mathrm{yr})\end{array}$ & Paleosols & $\begin{array}{l}\text { Thickness } \\
\text { depth (m) }\end{array}$ & $\begin{array}{l}\text { Thickness } \\
\text { for } \\
\text { MAR/SR }\end{array}$ & $\stackrel{S R}{S R}$ & $\begin{array}{c}\text { MAR } \\
(\mathrm{g} / \mathrm{m} 2 / \mathrm{yr})\end{array}$ & \\
\hline \multirow{2}{*}{$\begin{array}{l}\text { Top sequece - Top } \\
\text { G7 (G12) }\end{array}$} & & 15,000 & 23,220 & 8220 & 3.589 & 3.589 & 43.66 & 720 & & 0.700 & 0.700 & 8.52 & 141 & & 4.450 & 4.450 & 54.14 & 893 & & 0.600 & 0.600 & 7.30 & 120 & \\
\hline & G7 (GI2) & 23,220 & 23,340 & 120 & 0.508 & & & & unit3 (GI2) & 0.200 & & & & $\mathrm{kc} 2=\mathrm{G} 7(\mathrm{GI} 2)$ & i2) 0.630 & & & & $\begin{array}{l}\text { unit } 4 / \mathrm{HLN} \\
(\mathrm{GI} 2)\end{array}$ & 0.350 & & & & \\
\hline \multirow{2}{*}{$\begin{array}{l}\text { Top G7 - Top G4 } \\
\text { (GI3) }\end{array}$} & & 23,340 & 27,540 & 4200 & 4.137 & 4.645 & 110.60 & 1825 & & 2.300 & 2.500 & 59.52 & 982 & & 5.130 & 5.760 & 137.14 & 2263 & & 2.100 & 2.450 & 58.33 & 963 & a \\
\hline & G4 (GI3) & $\begin{array}{l}27,540 \\
27,780\end{array}$ & $\begin{array}{l}27,780 \\
28,600\end{array}$ & 240 & $\begin{array}{l}0.411 \\
1.113\end{array}$ & 1.524 & 185.85 & 3067 & unit5a (GI3) & $\begin{array}{l}0.300 \\
0.300\end{array}$ & 0.600 & 73.17 & 1207 & $\mathrm{~kb} 2=\mathrm{G} 4(\mathrm{GI} 3)$ & i3) $\begin{array}{r}0.510 \\
1.380\end{array}$ & 1.890 & 230.49 & 3803 & unit6 (GI3) & $\begin{array}{l}0.300 \\
0.750\end{array}$ & 1.050 & 128.05 & 2113 & \\
\hline $\begin{array}{l}\text { Top G4 - Top G3 } \\
\text { (GI4) }\end{array}$ & G3 (GI4) & 28,600 & 28.900 & 300 & 0.387 & & & & unit5c (GI4) & 0.250 & & & & $\mathrm{kA} 4=\mathrm{G} 3(\mathrm{G} / 4)$ & ;i4) $\quad 0.480$ & & & & unit8 (GI4) & 0.350 & & & & \\
\hline \multirow{2}{*}{$\begin{array}{l}\text { Top G3 - Top G2b } \\
\text { (G15) }\end{array}$} & & 28,900 & 32,040 & 3140 & 2.306 & 2.694 & 85.80 & 1416 & & 0.600 & 0.850 & 27.07 & 447 & & 2.860 & 3.340 & 106.37 & 1755 & & 1.400 & 1.750 & 55.73 & 920 & b \\
\hline & G2b (GI5) & 32,040 & 32,500 & 460 & 0.290 & & & & $\begin{array}{l}\text { unit7=HC6 } \\
\text { (GI5) }\end{array}$ & 0.200 & & & & $\mathrm{hc} 6=\mathrm{G} 2 \mathrm{~b}(\mathrm{Gl}$ & GI5) 0.360 & & & & unit10b (GI5) & 1.650 & & & & \\
\hline \multirow{2}{*}{$\begin{array}{l}\text { Top G2b - Top G2a } \\
\text { (GI6) }\end{array}$} & & 32,500 & 33,360 & 860 & 0.492 & 0.782 & 90.93 & 1500 & & 0.200 & 0.400 & 46.51 & 767 & & 0.610 & 0.970 & 112.79 & 1861 & & 0.100 & 1.750 & 203.49 & 3358 & \\
\hline & G2a (GI6) & 33,360 & 33,740 & 380 & 0.444 & & & & ? & & & & & $\mathrm{hc} 4=\mathrm{G} 2 \mathrm{a}(\mathrm{GI}$ & GI6) 0.550 & & & & unit12 (GI6) & 0.050 & & & & \\
\hline \multirow{2}{*}{$\begin{array}{l}\text { Top G2a - Top G1b } \\
\text { (G17) }\end{array}$} & & 33,740 & 34,740 & 1000 & 0.831 & 1.274 & 127.40 & 2102 & & & & & & & 1.030 & 1.580 & 158.00 & 2607 & & 0.350 & 0.400 & 40.00 & 660 & \\
\hline & G1b (G7) & 34,740 & 35,480 & 740 & 0.145 & & & & ? & & & & & gley (G7) & 0.180 & & & & unitX (G7) & 0.100 & & & & \\
\hline \multirow{2}{*}{$\begin{array}{l}\text { Top G1b - Top LB } \\
\text { (GI8) }\end{array}$} & & 35,480 & 36,580 & 1100 & 0.363 & 0.508 & 46.18 & 762 & & & & & & & 0.450 & 0.630 & 57.27 & 945 & & 0.000 & & & & \\
\hline & LB (GI8) & 36,580 & 38,220 & 1640 & 0.452 & & & & $\begin{array}{l}\text { unit8=LB } \\
\text { (GI8) }\end{array}$ & 0.500 & & & & $\mathrm{vxs}=\mathrm{LB}(\mathrm{GI})$ & 0.560 & & & & $\begin{array}{l}\text { unit14=LB } \\
\text { (GI8) }\end{array}$ & 0.650 & & & & \\
\hline \multicolumn{2}{|c|}{ Reference strati (Nussloch) } & \multicolumn{3}{|c|}{ NGRIP (Greenland) } & \multicolumn{4}{|c|}{ Nussloch (Germany) } & \multicolumn{5}{|c|}{ Dolni Vestonice (Czech Republic) } & \multicolumn{5}{|c|}{ Zlota (Poland) } & \multicolumn{5}{|c|}{ Stayky (Ukraine) } & Bond \\
\hline $\begin{array}{c}\text { Eolian } \\
\text { sedimentation }\end{array}$ & Paleosols & $\begin{array}{l}\text { Age } \\
\text { end } \\
(\mathrm{yr})\end{array}$ & $\begin{array}{l}\text { Age } \\
\text { start } \\
(\mathrm{yr})\end{array}$ & $\begin{array}{c}\text { Duration } \\
\text { (yr) }\end{array}$ & $\begin{array}{l}\text { Thickness } \\
\text { depth }(\mathrm{m})\end{array}$ & $\begin{array}{l}\text { Thickness } \\
\text { for } \\
\text { MAR/SR }\end{array}$ & s $\begin{array}{c}\text { SR } \\
(\mathrm{cm} / \mathrm{kyr})\end{array}$ & $\begin{array}{c}\text { MAR } \\
(\mathrm{g} / \mathrm{m} 2 / \mathrm{yr})\end{array}$ & Paleosols & $\begin{array}{l}\text { Thickness } \\
\text { depth (m) }\end{array}$ & $\begin{array}{l}\text { s. Thickness } \\
\text { ) for } \\
\text { MAR/SR }\end{array}$ & $\begin{array}{l}\mathrm{s} \\
(\mathrm{cm} / \mathrm{kgyr})\end{array}$ & $\begin{array}{c}\text { MAR } \\
(\mathrm{g} / \mathrm{m} 2 / \mathrm{yr})\end{array}$ & Paleosols $\frac{T}{d}$ & $\begin{array}{l}\text { Thickness } \\
\text { depth (m) }\end{array}$ & $\begin{array}{l}\text { Thickness } \\
\text { for } \\
\text { MAR/SR }\end{array}$ & $\begin{array}{c}\mathrm{SR} \\
(\mathrm{cm} / \mathrm{kyr})\end{array}$ & $\begin{array}{c}\text { MAR } \\
(\mathrm{g} / \mathrm{m} 2 / \mathrm{yr})\end{array}$ & Paleosols & $\begin{array}{l}\text { Thickness } \\
\text { depth (m) }\end{array}$ & $\begin{array}{l}\text { Thickness } \\
\text { for } \\
\text { MAR/SR }\end{array}$ & $\begin{array}{c}\mathrm{SR} \\
(\mathrm{cm} / \mathrm{kyr})\end{array}$ & $\begin{array}{c}\mathrm{MAR} \\
(\mathrm{g} / \mathrm{m} 2 / \mathrm{yr})\end{array}$ & \\
\hline \multirow{2}{*}{$\begin{array}{l}\text { Top sequece - Top } \\
\text { G7 (GI2) }\end{array}$} & & 15,000 & 23,220 & 8220 & 3.589 & 3.589 & 43.66 & 720 & & 0.900 & 0.900 & 10.94 & 181 & & 1.957 & 1.957 & 23.81 & 393 & & 1.500 & 1.500 & 18.25 & 301 & \\
\hline & G7 (GI2) & 23,220 & 23,340 & 120 & 0.508 & & & & $\begin{array}{l}\text { pseudogley } \\
=\mathrm{G} 7(\mathrm{GI} 2)\end{array}$ & 0,150 & & & & $\mathrm{G} 7(\mathrm{GI} 2) \quad 0$ & 0,277 & & & & "TL2 (GI2) & 0,100 & & & & \\
\hline \multirow{2}{*}{$\begin{array}{l}\text { Top G7 - Top G4 } \\
\text { (GI3) }\end{array}$} & & 23,340 & 27,540 & 4200 & 4,137 & 4,645 & 110,60 & 1825 & & 4,800 & 4,950 & 117,85 & 1945 & & 1,851 & 2,128 & 50,66 & 836 & & 2,900 & 3,000 & 71,43 & 1179 & a \\
\hline & G4 (GI3) & 27,540 & 27,780 & 240 & 0,411 & & & & $\begin{array}{l}\text { unit3 } \\
=\mathrm{G} 4(\mathrm{GI} 3)\end{array}$ & 0,350 & & & & $\mathrm{G} 4(\mathrm{GI} 3)$ & 0.191 & & & & unit3DV (GI3) & 0.300 & & & & \\
\hline $\begin{array}{l}\text { Top G4 - Top G3 } \\
\text { (GI4) }\end{array}$ & & 27,780 & 28,600 & 820 & 1.113 & 1.524 & 185.85 & 3067 & & & & & & & 0.745 & 0.936 & 114.17 & 1884 & & 0.550 & 0.850 & 103.66 & 1710 & \\
\hline & G3 (GI4) & 28,600 & 28,900 & 300 & 0.387 & & & & G3 (GI4) & & & & & G3 (GI4) 0 & 0.255 & & & & TL4 (GI4) & 0.150 & & & & \\
\hline $\begin{array}{l}\text { Top G3 - Top G2b } \\
\text { (GI5) }\end{array}$ & & 28,900 & 32,040 & 3140 & 2.306 & 2.694 & 85.80 & 1416 & & & & & & & 0.426 & 0.681 & 21.68 & 358 & & 0.475 & 0.625 & 19.90 & 328 & b \\
\hline & $\mathrm{G} 2 \mathrm{~b}(\mathrm{GIS})$ & 32,040 & 32,500 & 460 & 0.290 & & & & G2b (GI5) & & & & & G2b (GI5) 0 & 0.213 & & & & paleo6a-6b (GI5) & 0.325 & & & & \\
\hline $\begin{array}{l}\text { Top G2b - Top G2a } \\
\text { (G16) }\end{array}$ & & 32,500 & 33,360 & 860 & 0.492 & 0.782 & 90.93 & 1500 & & & & & & & 0.489 & 0.702 & 81.64 & 1347 & & 0.400 & 0.725 & 84.30 & 1391 & \\
\hline & G2a (GI6) & 33,360 & 33,740 & 380 & 0.444 & & & & G2a (GI6) & & & & & G2a (G16) 0 & 0.277 & & & & paleo6c (GI6) & 0.275 & & & & \\
\hline $\begin{array}{l}\text { Top G2a - Top G1b } \\
\text { (GI7) }\end{array}$ & & 33,740 & 34,740 & 1000 & 0.831 & 1.274 & 127.40 & 2102 & & & & & & & 0.915 & 1.191 & 119.15 & 1966 & & 0.525 & 0.800 & 80.00 & 1320 & \\
\hline & $\mathrm{G} 1 \mathrm{~b}(\mathrm{G} 7)$ & $\begin{array}{l}34,740 \\
33540\end{array}$ & 35,480 & $\begin{array}{l}740 \\
1100\end{array}$ & $\begin{array}{l}0.145 \\
0.363\end{array}$ & 0.508 & 46.18 & 762 & G1b (G7) & & & & & G1b (G7) 0 & $\begin{array}{l}0.255 \\
0.553\end{array}$ & 0.809 & 7350 & $\mathrm{pa}$ & paleo7-8 (G7) & 0.350 & 0750 & 6818 & 1125 & \\
\hline & LB (GI8) & 36,580 & 38,220 & 1640 & 0.452 & & & & unit $4=$ =LB & 0.200 & & & & LB (GI8) & 0.638 & & & & Vytachiv $=$ LB & 0.300 & & & & \\
\hline
\end{tabular}




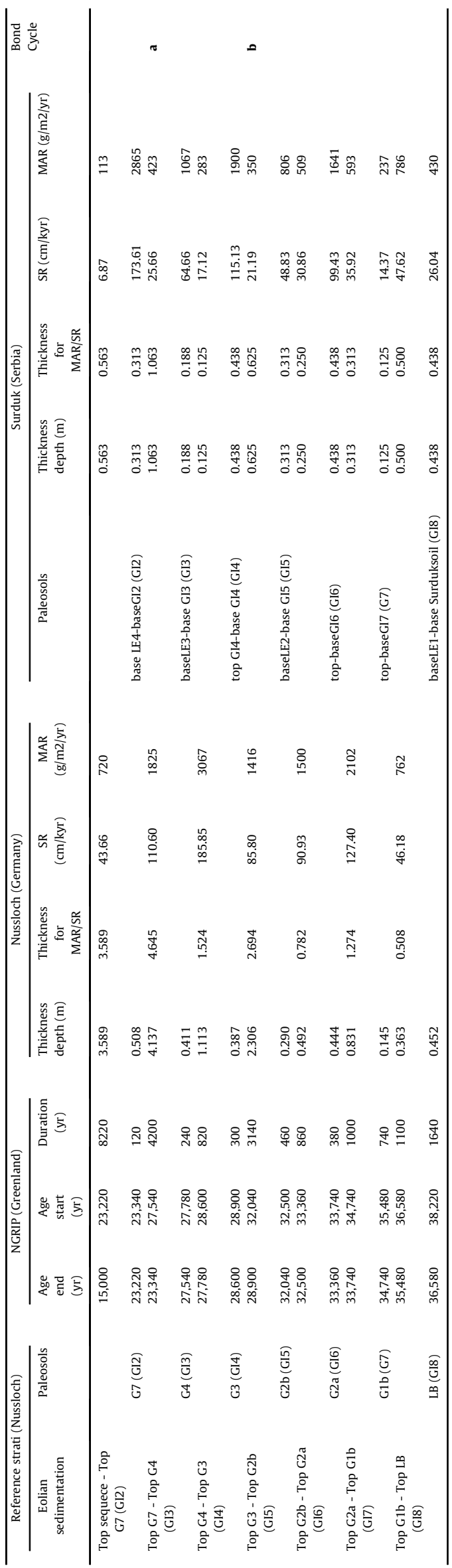

BCA indicates the highest MAR in maximum and minimum ranges (3803-1710 g/m $/ \mathrm{m}^{2} / \mathrm{yr}$ and 2263-836 g/m $/ \mathrm{m}^{2} / \mathrm{yr}$ ), than BCB (3358-1391 g/m $/ \mathrm{m}^{2} / \mathrm{yr}$ and $945-328 \mathrm{~g} / \mathrm{m}^{2} / \mathrm{yr}$ ) (Fig. 4; Table 2). The MAR estimated from sequences located at $50^{\circ} \mathrm{N}$, especially in central and eastern Europe do have a similar pattern compared to those calculated from the Carpathian Basin, at least in Surduk, Serbia. Reconstructions of bulk MARs are in agreement with former estimates from Dunaszekcso in southern Hungary (Ujvari et al., 2017) indicating average values of $1494 \mathrm{~g} / \mathrm{m}^{2} / \mathrm{yr}$ and $717 \mathrm{~g} / \mathrm{m}^{2} / \mathrm{yr}$ for BCA and BCB respectively. As already reported from our SR estimates, a west-east gradient appears along the longitudinal transect with the highest values from west-European sequences (Fig. 4).

Our finding appears to contradict with previous estimates for LGM MAR in Europe, and in some cases for the same sites. Frechen et al. (2003) indicate $3135-1467 \mathrm{~g} / \mathrm{m}^{2} / \mathrm{yr}$ for Harmignies, $453 \mathrm{~g} / \mathrm{m}^{2} /$ $\mathrm{yr}$ for Remicourt, 6129-1213 g/m²/yr for Nussloch and 1100-754 g/ $\mathrm{m}^{2} / \mathrm{yr}$ for Dolni Vestonice for the $28-18 \mathrm{ka}$ interval corresponding to BCA. Although the estimates for Harmignies by Frechen et al. (2003) are close to our reconstructions, this is not at all the case for the other sites. Such differences could be attributed to different age models of these loess records in our case by applying the complex relationship of the paleosol/pedogenic horizon constraint. Albani et al. (2014) estimated bulk flux of $242 \mathrm{~g} / \mathrm{m}^{2} / \mathrm{yr}$ for St. Pierrelès-Elbeuf, $412 \mathrm{~g} / \mathrm{m}^{2} / \mathrm{yr}$ for Harmignies, $560 \mathrm{~g} / \mathrm{m}^{2} / \mathrm{yr}$ for Remicourt, $2114 \mathrm{~g} / \mathrm{m}^{2} / \mathrm{yr}$ for Nussloch and $758 \mathrm{~g} / \mathrm{m}^{2} / \mathrm{yr}$ for Dolni Vestonice for the 25-12ka interval. Based on a different timescale, a similar conclusion could be proposed for these reconstructions, though these MARs seem underestimated compared to ours.

\section{Comparison with Chinese loess sequences}

Millennial variations are identified from high-sedimentationrate loess sequences located on the northwestern Chinese loess Plateau, but without any paleosol identified in the LCC as observed in Europe (Sun et al., 2012). Based on numerous OSL dates of the Gulang and Jingyuan sequences, Sun et al. (2012) suggested that variations of the East Asian winter monsoon as inferred from loess grain size are well correlated with millennial climate changes recorded in the Chinese Hulu/Wulu speleothems and the Greenland ice core. Generally, GSs correspond to coarser loess units whereas, GIs are characterized by increased contribution of finer dust materials originated from northern Chinese deserts, mostly the neighboring Tengger Desert (Fig. 5). This result supports the previous observations performed all over the Chinese Loess Plateau (e.g. Ding et al., 1999; Porter and An, 1995; Yang and Ding, 2014). Therefore, our methodology used for European sequences about the dust deposition does not apply to these records, which are interpreted as having dust deposition also during GIs, as observed also in modern conditions through continental dust storms (Sun et al., 2001).

To compare our results from European sequences with Chinese loess records, we have used the correspondences between the published age model and the measured depth of the Chinese equivalents of the Greenland GSs and GIs in Gulang and Jingyuan loess sequences from Sun et al. (2012), and the time boundaries of the GSs and GIs from the Greenland ice-cores as defined by Rasmussen et al. (2014). Strong similarity in millennial-scale changes in mean grain size variations from the Gulang and Jingyuan loess series and $\delta^{18} \mathrm{O}$ record from the Hulu Cave and Greenland ice core yields precise correlation of these DO cycles during the 60-23 ka (Rasmussen et al., 2014; Sun et al., 2012; Wang et al., 2001). Such correlation was lately extended to the last two climatic cycles by a 249 ka stack of eight loess grain size records from northern China (Yang and Ding, 2014), Bulk SR and MAR of 


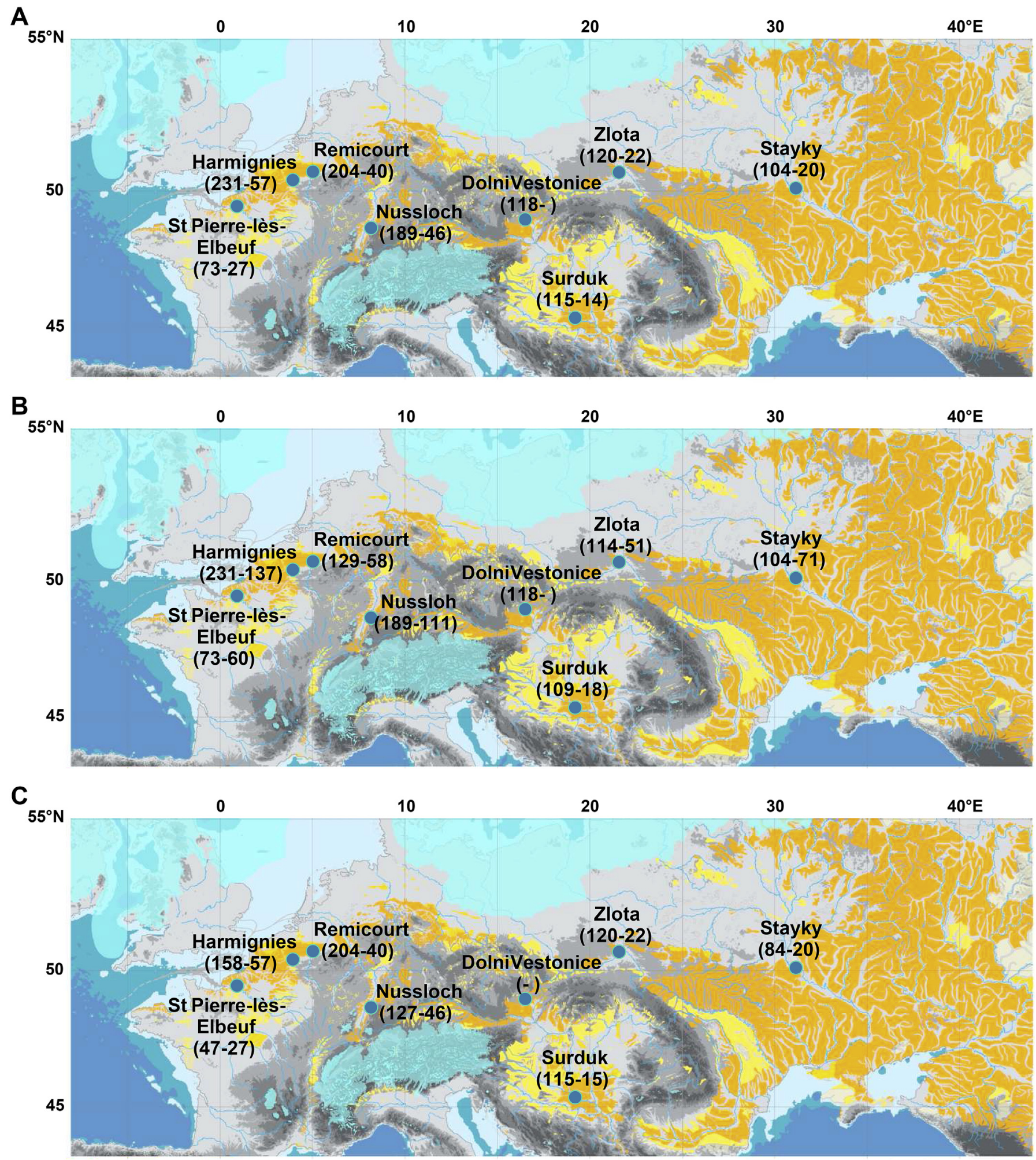

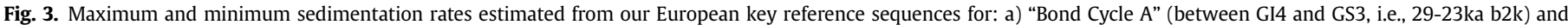

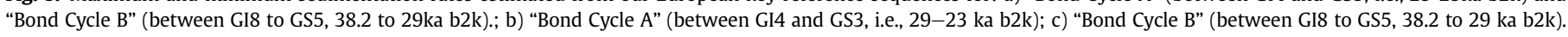
Same caption as in Fig. 1. 


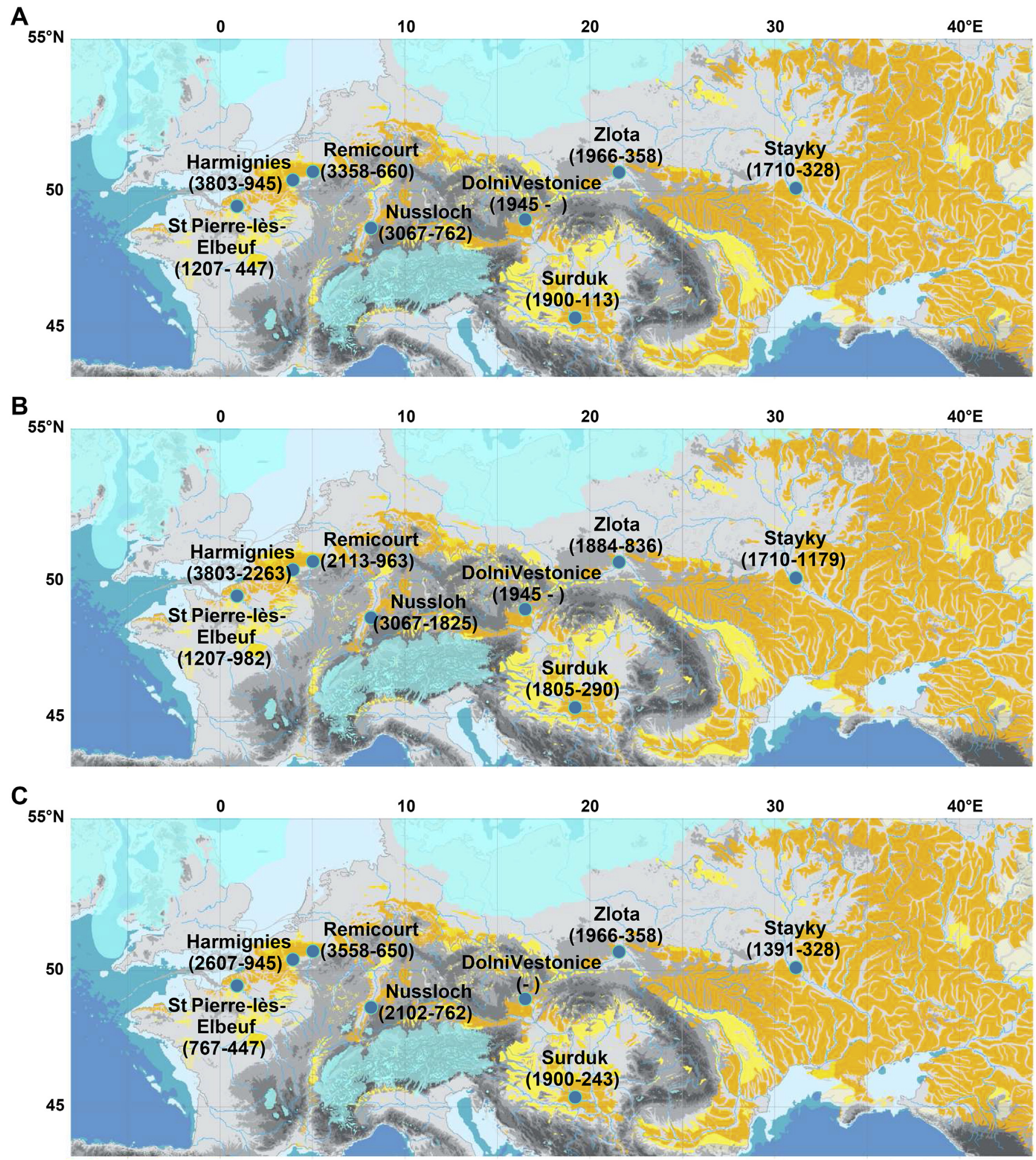

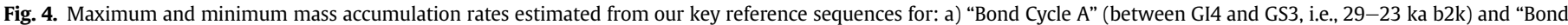
Cycle B" (between GI8 to GS5, 38.2 to 29 ka b2k); b) "Bond Cycle A" (between GI4 and GS3, i.e., 29-23 ka b2k); c) "Bond Cycle B" (between GI8 to GS5, 38.2-29 ka b2k). 


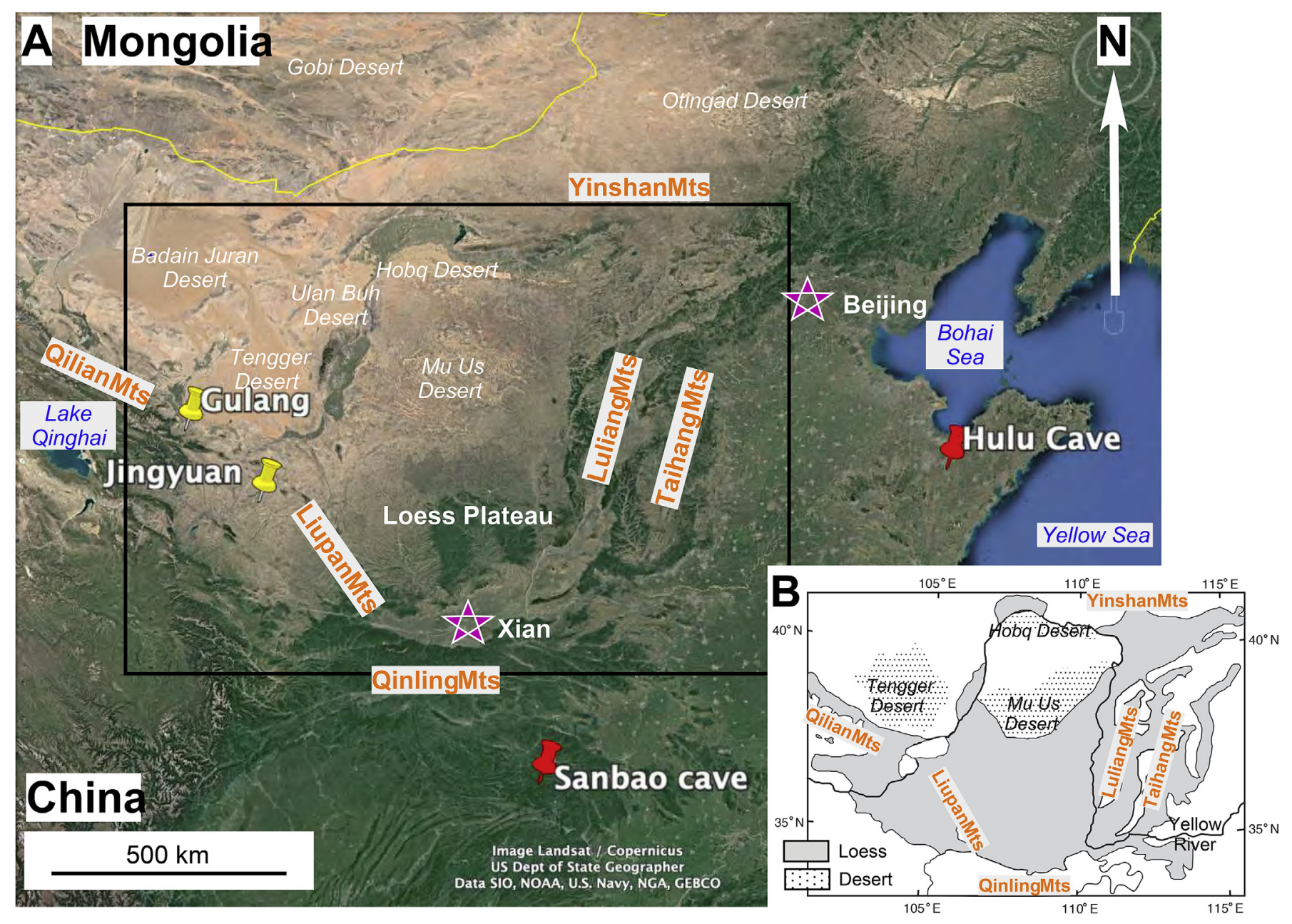

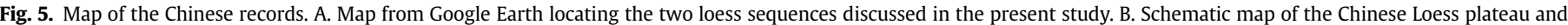
the surrounding main deserts. (from (Hao et al., 2010) modified).

these two Chinese sequences are therefore estimated to compare with those from our European sequences, during $\mathrm{BCA}$ and $\mathrm{BCB}$ (Fig. 6; Table 3).

SRs at Gulang and Jungyuan always show values lower than our European sequences, consistent with those obtained for St. Pierrelès-Elbeuf which is less than $100 \mathrm{~cm} / \mathrm{kyr}$. Interestingly, Jingyuan always shows higher values than Gulang, but also coarser material according to Sun et al. (2012). This result probably relates to the location of the two sites with regard to the dust source. Gulang is protected by the mountain range bordering westwards the Tengger desert while Jingyuan is very close to the Yellow River where coarser material can be easily transported by the East Asian monsoon wind. Estimated MARs at Gulang and Jingyuan are lower by a factor of 2-3 than those computed for the European sequences for BCA and BCB. Jingyuan shows higher values than Gulang, probably because of its downwind location from the proximal dust sources The Chinese loess plateau is worldwide known as a remarkable record of the Tertiary and Quaternary eolian deposits, yielding a unique continental archive to document changes in East Asian paleomonsoon and dust deposition over the last $25 \mathrm{Ma}$ (Guo et al., 2002; Qiang et al., 2011). Estimated MARs from loess sequences in the central Chinese Loess Plateau, ranging between 111.5 and $758.9 \mathrm{~g}: \mathrm{m}^{2} / \mathrm{yr}$ during the last glacial maximum (An et al., 1991; Liu et al., 2019) are much lower than those from the northern western Chinese Loess Plateau.

Our comparison between European and Chinese loess sequences shows that the dust loading of the atmosphere, was much higher in Europe than in China by a factor of 3, at least during BCA and $\mathrm{BCB}$, and therefore during mostly the LGM, with the dustiest conditions of the last climate cycle. However, this difference in dust loading between the two regions does not imply that the frequency of the dust events, transport and deposition was lower in China. Rather, as continuous dust deposition prevailed in Asia, one could assume that dust storms were more frequent than in Europe, similar to today between the Gobi and Taklamakan deserts showing higher frequencies of dust storm for the former but a high dust amount emitted for the latter (Laurent et al., 2006). The difference in MAR is likely attributed to different source-to-sink dust systems between Europe and East Asia, since Asian dust is mainly transported from distant provisional sources such as the Gobi and sandy deserts in northwest China and southern Mongolia (Sun et al., 2020). 

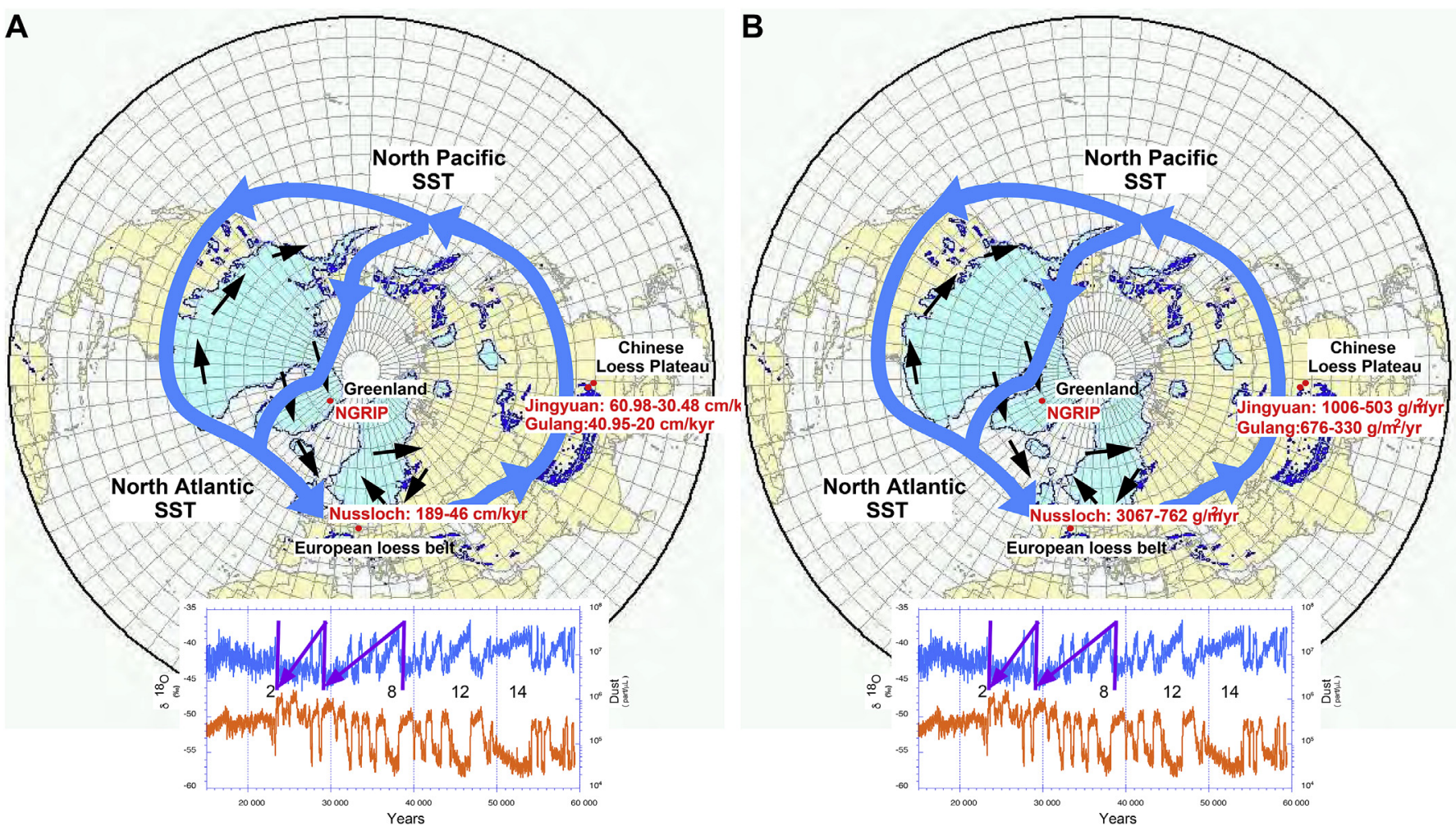

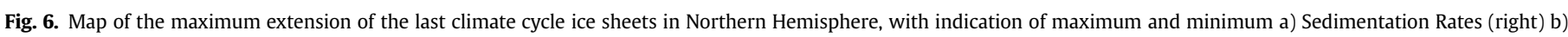

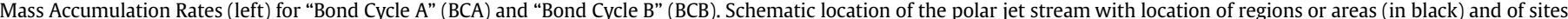

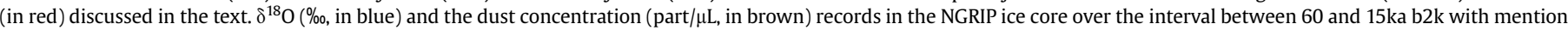

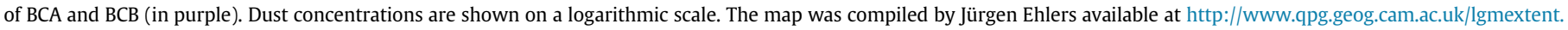
html. . (For interpretation of the references to colour in this figure legend, the reader is referred to the Web version of this article.)

\section{Comparison with Earth System model estimates}

When modeling the LGM dust deposition worldwide, Mahowald et al., 2006, 2011 indicated much lower values for European loess deposits than our estimates. Indeed, in the region corresponding to Harmignies, Remicourt and St. Pierre-lès-Elbeuf, Mahowald et al. (Mahowald et al, 2006, 2011) indicate MAR of $5-10 \mathrm{~g} / \mathrm{m}^{2} / \mathrm{yr}$, of $10-20 \mathrm{~g} / \mathrm{m}^{2} / \mathrm{yr}$ for the Nussloch area, $50-100 \mathrm{~g} / \mathrm{m}^{2} / \mathrm{yr}$ for the Zlota area, and finally $100-200 \mathrm{~g} / \mathrm{m}^{2} / \mathrm{yr}$ for the area including Dolni Vestonice, Stayky and Surduk. These estimates fit with other LGM deposition rates for Europe, proposed most recently by Albani et al. (2014) and Lambert et al. (2015) indicating MAR values higher than $50 \mathrm{~g} / \mathrm{m}^{2} / \mathrm{yr}$ (Kageyama et al., 2018). On the contrary, Hopcroft et al. (2015) rather indicated even lower deposition rates of about $1 \mathrm{~g} /$ $\mathrm{m}^{2} / \mathrm{yr}$ for the same region.

The differences between these model estimates and our reconstructions, which could be considered as significant, rely on the fact that models only consider the fine-grained mineral component of the dust deposits, up to about $10 \mu \mathrm{m}$. In contrast, our initial reconstructions were based on bulk material, which includes grain size categories coarser than the mineral aerosol size used in climate models. The assignment of every sample taken from the outcrop into four main grain-size classes allows a slightly different MAR computation, with particle sizes $<4.6 \mu \mathrm{m}$ for clays, between $4.6 \mu \mathrm{m}$ and $26 \mu \mathrm{m}$ for fine silts, between $26 \mu \mathrm{m}$ and $63 \mu \mathrm{m}$ for coarse silts and $>63 \mu \mathrm{m}$ for sand (Table 4 ). When taking into account the clay fraction, which is closer to the mineral aerosol size used by the models, Nussloch loess sequence yields, MAR varying between 181 and $126 \mathrm{~g} / \mathrm{m} 2 / \mathrm{yr}$ for BCA, and between 109 and $66 \mathrm{~g} / \mathrm{m}^{2} / \mathrm{yr}$ for BCB, which are values closer to the estimates from Albani et al. (2014) and Lambert et al. (2015) for the LGM encompassed in BCA. Interestingly, considering the clay fraction, the MAR estimates for BCA in Dolni Vestonice and Stayky are higher than in Nussloch, between 240 and $145 \mathrm{~g} / \mathrm{m} 2 / \mathrm{yr}$, in agreement with the average $<10 \mu \mathrm{m}$ fraction flux estimated for BCA and BCB, 269 and $157 \mathrm{~g} / \mathrm{m} 2 / \mathrm{yr}$ respectively, from southern Hungary (Ujvari et al., 2017), but also the MAR range for the area grouping these three sites by Albani et al. (2016).

Using the grain size results for Nussloch, Dolni Vestonice and Stayky, the respective contributions of the four main categories to the bulk MARs can be evaluated (Table 4). Although the highest values are obtained for BCA and for the coarse silt fraction, 


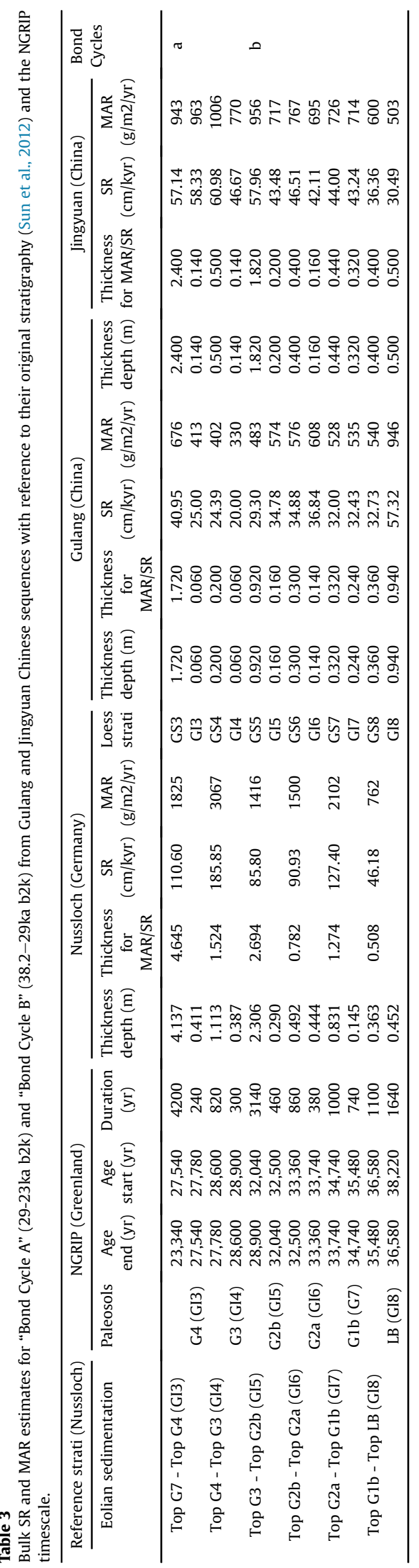

$26 \mu \mathrm{m}-63 \mu \mathrm{m}$, no particular pattern prevails. BCB reveals lower, but still high values for Nussloch compared to the other sequences.

\section{Conclusions and outlook}

The Greenland Interstadials described in the Last Climate Cycle from ice core records have their equivalent in the European loess sequences as paleosols, tundra gleys or embryonic soils. These loess series also show a complicated evolution in time duration as the pedogenic units developed downward into the top of the eolian units when dust deposition stopped. The observation leads to a reconsideration of the time interval corresponding to the real dust deposition in terms of the thickness of the observed loess units, and that of the overlying paleosols in contrast to other records of continuous deposition.

We applied this new stratigraphical and chronological concept to the Nussloch sequence as a reference record of the LCC, and then compared this record to other European high-resolution loess sequences located at about $50^{\circ} \mathrm{N}$. By correlating with the Greenland ice core timescale, we assigned ages to the continental equivalents of GSs and GIs. Doing so, we have grouped GS and GI European equivalents according to the definition of the Bond Cycles, which represent long-term cooling cycles gathering several interstadialstadial cycles. Our study shows that two Bond Cycles are well preserved in the European loess sequences, namely BCA between GI4 and GS3 (i.e., 29-23ka b2k), and BCB between GI8 to GS5 (i.e. $38.2-29 \mathrm{ka} b 2 \mathrm{k}$ ), BCA shows the highest SRs and MARs in all the studied sequences. Due to the new methodology we applied to the age model, our estimates differ from previous investigations which show relatively lower values. Our study also shows that a longitudinal gradient in the bulk estimates prevailed with the highest values in western European sequences and that the dustiest conditions were not necessarily linked to Heinrich stadials ending all Bond cycles.

Comparison of European loess records with two Chinese loess sequences reveals that while millennial scale variability is evident in these loess profiles, no pedogenic unit is punctuating the rather continuous dust deposition on the Chinese Loess Plateau. The comparison between the Chinese and the European sequences shows that bulk SR and MAR estimates are much higher in Europe than in China, indicating that during the last Glacial Maximum stadials, the atmosphere may have been dustiest in western Eurasia compared to East Asia. However, proposing a dustier Europe does not imply that the dust storm frequencies were higher in Europe but rather the dust amount emitted during one particular dust storm in Europe was higher than in Asia.

A final validation of our study has been performed by a comparison of our estimates with dust deposition rates computed from Earth System models. We assigned grain size results of the loess samples into four main categories for better comparison with the model results. The clay-sized fractions of the loess samples is close to the grain size of mineral aerosol considered by Earth System models. The comparison between our MAR estimations and the model results shows similar values, yielding a promising result for further model-data assimilations. 
Table 4

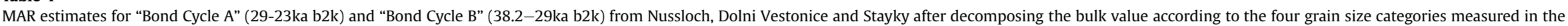
sediment samples taken on the studied outcrops. Data from Antoine et al. (2009b), Rousseau et al. (2011), Antoine et al. (2013).

\begin{tabular}{|c|c|c|c|c|c|c|c|c|c|c|c|}
\hline \multirow[t]{2}{*}{ Nussloch } & \multirow{2}{*}{$\begin{array}{l}\text { MAR bulk } \\
\text { (g/m2/yr) }\end{array}$} & \multicolumn{4}{|c|}{ Mean grain size } & \multicolumn{4}{|c|}{ MAR by grain size } & \multirow[t]{2}{*}{ Eolian sedimentation } & \multirow{2}{*}{$\begin{array}{l}\text { Bond } \\
\text { Cycle }\end{array}$} \\
\hline & & $\begin{array}{l}\text { M clay } \\
(<4.6 \mu \mathrm{m}) \%\end{array}$ & $\begin{array}{l}\text { M fine silt } \\
(4.6-26 \mu \mathrm{m}) \%\end{array}$ & $\begin{array}{l}\text { M coarse silt } \\
(26-63 \mu \mathrm{m}) \%\end{array}$ & $\begin{array}{l}\text { M sand } \\
(>63 \mu \mathrm{m}) \%\end{array}$ & $\begin{array}{l}\text { MAR clay } \\
\text { (g/m2/yr) }\end{array}$ & $\begin{array}{l}\text { MAR fine silt } \\
(\mathrm{g} / \mathrm{m} 2 / \mathrm{yr})\end{array}$ & $\begin{array}{l}\text { MAR coarse } \\
\text { silt }(\mathrm{g} / \mathrm{m} 2 / \mathrm{yr})\end{array}$ & $\begin{array}{l}\text { MAR sand } \\
(\mathrm{g} / \mathrm{m} 2 / \mathrm{yr})\end{array}$ & & \\
\hline & 720 & 9.54 & 27.95 & 47.81 & 14.71 & 69 & 201 & 344 & 106 & between GI2 \& top & \\
\hline G4 (GI3) & 1625 & 7.72 & 27.44 & 50.59 & 14.25 & 126 & 446 & 822 & 232 & between GI2 \& GI3 & $\mathbf{a}$ \\
\hline G3 (GI4) & 2239 & 8.10 & 26.97 & 50.77 & 14.16 & 181 & 604 & 1137 & 317 & between GI3 \& GI4 & \\
\hline G2b (GI5) & 1212 & 7.41 & 26.96 & 52.39 & 13.24 & 90 & 327 & 635 & 160 & between GI4 \& GI5 & b \\
\hline G2a (GI6) & 944 & 9.12 & 35.16 & 47.40 & 8.33 & 86 & 332 & 447 & 79 & between GI5 \& GI6 & \\
\hline G1b (G7) & 1371 & 7.92 & 34.16 & 47.16 & 10.76 & 109 & 468 & 646 & 148 & between GI6 \& GI7 & \\
\hline LB (GI8) & 544 & 12.12 & 37.64 & 40.74 & 9.50 & 66 & 205 & 222 & 52 & between GI7 \& GI8 & \\
\hline
\end{tabular}

\begin{tabular}{|c|c|c|c|c|c|c|c|c|c|c|c|}
\hline & 321 & 12.83 & 29.52 & 40.39 & 17.26 & 41 & 95 & 130 & 55 & between GI2 \& top & \\
\hline & 1139 & 12.75 & 33.03 & 40.11 & 14.11 & 145 & 376 & 457 & 161 & between GI2 \& GI3 & $\mathbf{a}$ \\
\hline 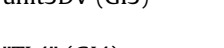 & 1107 & 15.04 & 36.36 & 34.96 & 13.64 & 166 & 402 & 387 & 151 & between GI3 \& GI4 & \\
\hline $\begin{array}{l}\text { "TL4" (GI4) } \\
\text { paleo6a-6b (GI5) }\end{array}$ & 250 & 17.04 & 36.70 & 33.56 & 12.70 & 43 & 92 & 84 & 32 & between GI4 \& GI5 & b \\
\hline paleo6c (GI6) & 767 & 17.05 & 37.05 & 34.3 & 11.6 & 131 & 284 & 263 & 89 & between GI5 \& GI6 & \\
\hline paleo7-8 (G7) & 866 & 19.26 & 42.40 & 28.14 & 10.20 & 167 & 367 & 244 & 88 & between GI6 \& GI7 & \\
\hline Vytachiv $=$ LB (GI8) & 600 & 25.10 & 43.70 & 18.50 & 12.70 & 151 & 262 & 111 & 76 & between GI7 \& GI8 & \\
\hline
\end{tabular}

\section{Dolni Vestonice}

\begin{tabular}{|c|c|c|c|c|c|c|c|c|c|c|c|}
\hline pseudogley = G7 (GI2) & 181 & 12.83 & 29.52 & 40.39 & 17.26 & 23 & 53 & 73 & 31 & Top seq - Top pseudogley (GI2) & \\
\hline unit3 = G4 (GI3) & 1886 & 12.75 & 33.03 & 40.11 & 14.11 & 240 & 623 & 756 & 266 & $\begin{array}{l}\text { Top pseudogley - Top unit3 (GI3) } \\
\text { Top G4 - Top G3 (GI4) }\end{array}$ & $\mathbf{a}$ \\
\hline
\end{tabular}




\section{Author Contributions}

D.-D. R. designed and performed research; D.-D. R., P. A., and Y. S. analyzed data; and D.-D. R. wrote the paper with contributions from P. A. and Y. S.

\section{Data and materials availability}

Correspondence and material request should be addressed to D.-D. R.

\section{Declaration of Competing interest}

The authors declare that they have no known competing financial interests or personal relationships that could have appeared to influence the work reported in this paper.

\section{Acknowledgements}

The comments from Peter Clark on a preliminary version of this paper were very useful and welcomed. We would like to thank Jef Vandenberghe and an anonymous reviewer for their useful comments which contributed to improve the first version of this paper. Joyce Gavin helped in the editing of the revised version of the manuscript. These results were presented during EGU general assembly 2018 and benefited from the audience comments. DDR is also funded by the European Union's Horizon 2020 research and innovation program (TiPES: Tipping Points in Earth System (grant no. 820970)). This is an LDEO contribution \#8460, and TiPES contribution \#71.

\section{Appendix A. Supplementary data}

Supplementary data to this article can be found online at https://doi.org/10.1016/j.quascirev.2020.106775.

\section{References}

Adolphi, F., Ramsey, C.B., Erhardt, T., Edwards, R.L., Cheng, H., Turney, C.S.M., Cooper, A., Svensson, A., Rasmussen, S.O., Fischer, H., Muscheler, R., 2018. Connecting the Greenland ice-core and U/Th timescales via cosmogenic radionuclides: testing the synchroneity of Dansgaard-Oeschger events. Clim. Past $14,1755-1781$

Albani, S., Mahowald, N.M., Murphy, L.N., Raiswell, R., Moore, J.K., Anderson, R.F., McGee, D., Bradtmiller, L.I., Delmonte, B., Hesse, P.P., Mayewski, P.A., 2016. Paleodust variability since the Last Glacial Maximum and implications for iron inputs to the ocean. Geophys. Res. Lett. 43, 3944-3954.

Albani, S., Mahowald, N.M., Perry, A.T., Scanza, R.A., Zender, C.S., Heavens, N.G., Maggi, V., Kok, J.F., Otto-Bliesner, B.L., 2014. Improved dust representation in the community atmosphere model. J. Adv. Model. Earth Syst. 6, 541-570.

Alley, R.B., 1998. Palaeoclimatology - icing the North atlantic. Nature 392, 335.

Alley, R.B., Clark, P.U., Keigwin, L.D., Webb, R.S., 1999. Making sense of millenialscale climate change. In: Clark, P.U., Webb, R., Keigwin, L.D. (Eds.), Mechanisms of Global Climate Change at Millenial Time Scales. AGU, Snowbird, Utah, pp. 385-394.

Antoine, P. Coutard, S., Guerin, G., Deschodt, L, Goval, E, Locht, J.L, Paris, C., 2016. Upper pleistocene loess-palaeosol records from northern France in the European context: environmental background and dating of the middle palaeolithic. Quat. Int. 411, 4-24.

Antoine, P., Goval, E., Jamet, G., Coutard, S., Moine, O., Hérisson, D., Auguste, P., Guérin, G., Lagroix, F., Schmidt, E., Robert, V., Debenham, N., Meszner, S., Bahain, J.J., 2014. - the upper pleistocene loess sequences of havrincourt (Pasde-Calais, France). Stratigraphy, palaeoenvironments, geochronology and human occupations 25, 368.

Antoine, P., Rousseau, D.D., Degeai, J.P., Moine, O., Lagroix, F., kreutzer, S., Fuchs, M., Hatté, C., Gauthier, C., Svoboda, J., Lisa, L., 2013. High-resolution record of the environmental response to climatic variations during the Last InterglacialGlacial cycle in Central Europe: the loess-palaeosol sequence of Dolní Věstonice (Czech Republic). Quat. Sci. Rev. 67, 17-38.

Antoine, P., Rousseau, D.D., Fuchs, M., Hatte, C., Gauthier, C., Markovic, S.B., Jovanovic, M., Gaudenyi, T., Moine, O., Rossignol, J., 2009a. High-resolution record of the last climatic cycle in the southern Carpathian Basin (Surduk, Vojvodina, Serbia). Quat. Int. 198, 19-36.
Antoine, P., Rousseau, D.D., Lautridou, J.P., Hatté, C., 1999. Last interglacial-glacial climatic cycle in loess-paleosol successions of north-western France. Boreas $28,551-563$.

Antoine, P., Rousseau, D.D., Moine, O., Kunesch, S., Hatte, C., Lang, A., Tissoux, H., Zöller, L., 2009b. Rapid and cyclic aeolian deposition during the Last Glacial in European loess: a high-resolution record from Nussloch, Germany. Quat. Sci. Rev. 28, 2955-2973.

Antoine, P., Rousseau, D.D., Zöller, L., Lang, A., Munaut, A.V., Hatté, C., Fontugne, M., 2001. High-resolution record of the last interglacial-glacial cycle in the loess palaeosol sequences of Nussloch (Rhine Valley-Germany). Quat. Int. 76/77, 211-229.

Bard, E., Rostek, F., Turon, J.L., Gendreau, S., 2000. Hydrological impact of Heinrich events in the subtropical northeast atlantic. Science 289, 1321-1324.

Bibus, E., Frechen, M., Kösel, M., Rähle, W., 2007. Das jungpleistozäne Lössprofil von Nussloch (SW-Wand) im Aufschluss der Heidelberger Zement AG. Eiszeitalt. Ggw. 56, 227-255.

Boers, N., Ghil, M., Rousseau, D.D., 2018. Ocean circulation, ice shelf, and sea ice interactions explain Dansgaard-Oeschger cycles. Proc. Natl. Acad. Sci. U.S.A. 115, E11005-E11014.

Bond, G., Broecker, W., Johnsen, S., McManus, J., Labeyrie, L., Jouzel, J., Bonani, G., 1993. Correlations between climate records from North Atlantic sediments and Greenland ice. Nature 365, 143-147.

Broecker, W.S., 1994. Massive iceberg discharges as triggers for global climate change. Nature 372, 421-424.

Clark, P.U., Dyke, A.S., Shakun, J.D., Carlson, A.E., Clark, J., Wohlfarth, B., Mitrovica, J.X., Hostetler, S.W., McCabe, A.M., 2009. The last glacial maximum. Science 325, 710-714.

Clark, P.U., Hostetler, S.W., Pisias, N.G., Schmittner, A., Meissner, K.J., 2007. Mechanisms for an $\sim 7-\mathrm{kyr}$ climate and sea-level oscillation during marine isotope stage 3, 173, 209-246.

Ding, Z.L., Ren, J.Z., Yang, S.L., Liu, T.S., 1999. Climate instability during the penultimate glaciation: evidence from two high-resolution loess records, China. Journal of Geophysical Research-Solid Earth 104, 20123-20132.

Fleitmann, D., Cheng, H., Badertscher, S., Edwards, R.L., Mudelsee, M., Goektuerk, O.M., Fankhauser, A., Pickering, R., Raible, C.C., Matter, A., Kramers, J., Tuysuz, O., 2009. Timing and climatic impact of Greenland interstadials recorded in stalagmites from northern Turkey. Geophys. Res. Lett. 36.

Frechen, M., Oches, E.A., Kohfeld, K.E., 2003. Loess in europe-mass accumulation rates during the last glacial period. Quat. Sci. Rev. 22, 1835-1857.

Ganopolski, A., Rahmstorf, S., 2001. Rapid changes of glacial climate simulated in a coupled climate model. Nature 409, 153-158.

Grant, K.M., Rohling, E.J., Bar-Matthews, M., Ayalon, A., Medina-Elizalde, M., Ramsey, C.B., Satow, C., Roberts, A.P., 2012. Rapid coupling between ice volume and polar temperature over the past 150,000 years. Nature 491, 744-747.

Guo, Z.T., Ruddiman, W.F., Hao, Q.Z., Wu, H.B., Qiao, Y.S., Zhu, R.X., Peng, S.Z., Wei, J.J., Yuan, B.Y., Liu, T.S., 2002. Onset of Asian desertification by $22 \mathrm{Myr}$ ago inferred from loess deposits in China. Nature 416, 159-163.

Hao, Q.Z., Guo, Z.T., Qiao, Y.S., Xu, B., Oldfield, F., 2010. Geochemical evidence for the provenance of middle Pleistocene loess deposits in southern China. Quat. Sci. Rev. 29, 3317-3326.

Harrison, S.P., Sanchez Goñi, M.F., 2010. - Global Patterns of Vegetation Response to Millennial-Scale Variability and Rapid Climate Change during the Last Glacial Period, vol. 29, p. 2980.

Hatté, C., Fontugne, M., Rousseau, D.D., Antoine, P., Zöller, L., Tisnérat-Laborde, N., Bentaleb, I., 1998. $\delta 13 \mathrm{C}$ variations of loess organic matter as a record of the vegetation response to climatic changes during the Weichselian. Geology 26, 583-586.

Hopcroft, P.O., Valdes, P.J., Woodward, S., Joshi, M.M., 2015. Last glacial maximum radiative forcing from mineral dust aerosols in an Earth system model. Journal of Geophysical Research-Atmospheres 120, 8186-8205.

Kageyama, M., Braconnot, P., Harrison, S.P., Haywood, A.M., Jungclaus, J.H., OttoBliesner, B.L., Peterschmitt, J.Y., Abe-Ouchi, A., Albani, S., Bartlein, P.J., Brierley, C., Crucifix, M., Dolan, A., Fernandez-Donado, L., Fischer, H., Hopcroft, P.O., Ivanovic, R.F., Lambert, F., Lunt, D.J., Mahowald, N.M., Peltier, W.R., Phipps, S.J., Roche, D.M., Schmidt, G.A., Tarasov, L., Valdes, P.J., Zhang, Q., Zhou, T.J., 2018. The PMIP4 contribution to CMIP6-Part 1: overview and over-arching analysis plan. Geosci. Model Dev. (GMD) 11, 1033-1057.

Kindler, P., Guillevic, M., Baumgartner, M., Schwander, J., Landais, A. Leuenberger, M., 2014. Temperature reconstruction from 10 to 120 kyr b2k from the NGRIP ice core. Clim. Past 10, 887-902.

Kohfeld, K.E., Harrison, S.P., 2003. Glacial-interglacial changes in dust deposition on the Chinese Loess Plateau. Quat. Sci. Rev. 22, 1859-1878.

Kukla, G., 1970. Correlation between loesses and deep-sea sediments. GFF (Geol. Foren. Stockh. Forh.) 92, 148-180.

Kukla, G., Koci, A., 1972. End of the last interglacial in the loess record. Quat. Res. 2, $374-383$.

Lambert, F., Tagliabue, A., Shaffer, G., Lamy, F., Winckler, G., Farias, L., Gallardo, L., De Pol-Holz, R., 2015. Dust fluxes and iron fertilization in holocene and last glacial maximum climates. Geophys. Res. Lett. 42, 6014-6023.

Laurent, B., Marticorena, B., Bergametti, G., Mei, F., 2006. Modeling mineral dust emissions from Chinese and Mongolian deserts. Global Planet. Change 52, $121-141$.

Lautridou, J.P., 1985. Le cycle périglaciaire Pléistocène en Europe du Nord-Ouest et plus particulièrement en Normandie. Centre Géomorphologie Caen. Université Caen, Caen, p. 908. 
Lohmann, G., Butzin, M., Eissner, N., Shi, X., Stepanek, C., 2020. Abrupt climate and weather changes across time scales. Paleoceanography and Paleoclimatology 35.

Mahowald, N., Albani, S., Engelstaedter, S., Winckler, G., Goman, M., 2011. Model insight into glacial-interglacial paleodust records. Quat. Sci. Rev. 30, 832-854.

Mahowald, N.M., Muhs, D.R., Levis, S., Rasch, P.J., Yoshioka, M., Zender, C.S., Luo, C. 2006. Change in atmospheric mineral aerosols in response to climate: last glacial period, preindustrial, modern, and doubled carbon dioxide climates. Journal of Geophysical Research-Atmospheres 111.

Moine, O., Antoine, P., Hatte, C., Landais, A., Mathieu, J., Prud'homme, C., Rousseau, D.-D., 2017. The impact of Last Glacial climate variability in westEuropean loess revealed by radiocarbon dating of fossil earthworm granules. Proc. Natl. Acad. Sci. U.S.A. 114, 6209-6214.

Moska, P., Adamiec, G., Jary, Z., Bluszcz, A., Poreba, G., Piotrowska, N., Krawczyk, M., Skurzynski, J., 2018. Luminescence chronostratigraphy for the loess deposits in Zlota, Poland. Geochronometria 45, 44-55.

Moska, P., Jary, Z., Adamiec, G., Bluszcz, A., 2015. OSL chronostratigraphy of a loesspalaeosol sequence in Zlota using quartz and polymineral fine grains. Radiat. Meas. 81, 23-31.

Porter, S.C., An, Z.S., 1995. Correlation between climate events in the North Atlantic and China during the last glaciation. Nature 375, 305-308.

Pye, K., 1987. Aeolian Dust and Dust Deposits. Academic Press.

Pye, K., 1995. The nature, origin and accumulation of loess. Quat. Sci. Rev. 14, 653-657.

Pye, K., Zhou, L.P., 1989. Late pleistocene and holocene aeolian dust deposition in north China and the northwest pacific ocean. Palaeogeogr. Palaeoclimatol. Palaeoecol. 73, 11-23.

Qiang, X.K., An, Z.S., Song, Y.G., Chang, H., Sun, Y.B., Liu, W.G., Ao, H., Dong, J.B., Fu, C.F., Wu, F., Lu, F.Y., Cai, Y.J., Zhou, W.J., Cao, J.J., Xu, X.W., Ai, L., 2011. New eolian red clay sequence on the western Chinese Loess Plateau linked to onset of Asian desertification about 25 Ma ago. Sci. China Earth Sci. 54, 136-144.

Rasmussen, S.O., Bigler, M., Blockley, S.P., Blunier, T., Buchardt, S.L., Clausen, H.B., Cvijanovic, I., Dahl-Jensen, D., Johnsen, S.J., Fischer, H., Gkinis, V., Guillevic, M., Hoek, W.Z., Lowe, J.J., Pedro, J.B., Popp, T., Seierstad, I.K., Steffensen, J.P., Svensson, A.M., Vallelonga, P., Vinther, B.M., Walker, M.J.C., Wheatley, J.J., Winstrup, M., 2014. A stratigraphic framework for abrupt climatic changes during the Last Glacial period based on three synchronized Greenland ice-core records: refining and extending the INTIMATE event stratigraphy. Quat. Sci. Rev. 106, 14-28.

Rousseau, D.-D., Chauvel, C., Sima, A., Hatte, C., Lagroix, F., Antoine, P., Balkanski, Y., Fuchs, M., Mellett, C., Kageyama, M., Ramstein, G., Lang, A., 2014. European glacial dust deposits: geochemical constraints on atmospheric dust cycle modeling. Geophys. Res. Lett. 41, 7666-7674.

Rousseau, D.-D., Derbyshire, E., Antoine, P., Hatté, C., 2018. European Loess records. In: Elias, S. (Ed.), Reference Module in Earth Systems and Environmental Sciences. Elsevier, p. 17.

Rousseau, D.D., Antoine, P., Gerasimenko, N., Sima, A., Fuchs, M., Hatte, C., Moine, O., Zoeller, L., 2011. North Atlantic abrupt climatic events of the last glacial period recorded in Ukrainian loess deposits. Clim. Past 7, 221-234.

Rousseau, D.D., Antoine, P., Hatté, C., Lang, A., Zöller, L., Fontugne, M., Ben Othman, D., Luck, J.M., Moine, O., Labonne, M., Bentaleb, I., Jolly, D., 2002. Abrupt millennial climatic changes from Nussloch (Germany) upper weichselian eolian records during the last glaciation. Quat. Sci. Rev. 21, 1577-1582.

Rousseau, D.D., Boers, N., Sima, A., Svensson, A., Bigler, M., Lagroix, F., Taylor, S., Antoine, P., 2017a. (MIS3 \& 2) millennial oscillations in Greenland dust and Eurasian aeolian records - a paleosol perspective. Quat. Sci. Rev. 169, 99-113.

Rousseau, D.D., Sima, A., Antoine, P., Hatte, C., Lang, A., Zöller, L., 2007. Link between European and North Atlantic abrupt climate changes over the last glaciation. Geophys. Res. Lett. 34.

Rousseau, D.D., Svensson, A., Bigler, M., Sima, A., Steffensen, J.P., Boers, N., 2017b. Eurasian contribution to the last glacial dust cycle: how are loess sequences built? Clim. Past 13.

Sanchez Goñi, M.F., Harrison, S.P., 2010. Millennial-scale Climate Variability and Vegetation Changes during the Last Glacial: Concepts and Terminology, vol. 29, p. 2827.

Sanchez Goñi, M.F.S., Desprat, S., Daniau, A.L., Bassinot, F.C., Polanco-Martínez, J.M., Harrison, S.P., Allen, J.R.M., Scott Anderson, R., Behling, H., Bonnefille, R., Burjachs, F., Carrión, J.S., Cheddadi, R., Clark, J.S., Combourieu-Nebout, N., Mustaphi, C.J.C., Debusk, G.H., Dupont, L.M., Finch, J.M., Fletcher, W.J.,
Giardini, M., González, C., Gosling, W.D., Grigg, L.D., Grimm, E.C., Hayashi, R., Helmens, K., Heusser, L.E., Hill, T., Hope, G., Huntley, B., Igarashi, Y., Irino, T., Jacobs, B., Jiménez-Moreno, G., Kawai, S., Peter Kershaw, A., Kumon, F., Lawson, I.T., Ledru, M.P., Lézine, A.M., Mei Liew, P., Magri, D., Marchant, R., Margari, V., Mayle, F.E., Merna Mckenzie, G., Moss, P., Müller, S., Müller, U.C. Naughton, F., Newnham, R.M., Oba, T., Pérez-Obiol, R., Pini, R., Ravazzi, C. Roucoux, K.H., Rucina, S.M., Scott, L., Takahara, H., Tzedakis, P.C., Urrego, D.H., Van Geel, B., Guido Valencia, B., Vandergoes, M.J., Vincens, A., Whitlock, C.L., Willard, D.A., Yamamoto, M., 2017. - the ACER Pollen and Charcoal Database: A Global Resource to Document Vegetation and Fire Response to Abrupt Climate Changes during the Last Glacial Period, vol. 9, p. 695.

Sanchez-Goni, M.F., Landais, A., Fletcher, W.J., Naughton, F., Desprat, S., Duprat, J., 2008. Contrasting impacts of Dansgaard-Oeschger events over a western European latitudinal transect modulated by orbital parameters. Quat. Sci. Rev. 27, $1136-1151$.

Sima, A., Kageyama, M., Rousseau, D.D., Ramstein, G., Balkanski, Y., Antoine, P., Hatté, C., 2013. Modeling dust emission response to North Atlantic millennialscale climate variations from the perspective of East European MIS3 loess deposits. Clim. Past 9, 1385-1402.

Sima, A., Rousseau, D.D., Kageyama, M., Ramstein, G., Schulz, M., Balkanski, Y., Antoine, P., Dulac, F., Hatte, C., 2009. Imprint of North-Atlantic abrupt climate changes on western European loess deposits as viewed in a dust emission model. Quat. Sci. Rev. 28, 2851-2866.

Sun, J.M., Zhang, M.Y., Liu, T.S., 2001. Spatial and temporal characteristics of dust storms in China and its surrounding regions, 1960-1999: relations to source area and climate. Journal of Geophysical Research-Atmospheres 106 10325-10333.

Sun, Y., Clemens, S.C., Morrill, C., Lin, X., Wang, X., An, Z., 2012. Influence of Atlantic meridional overturning circulation on the East Asian winter monsoon. Nat. Geosci. 5, 46-49.

Taylor, S.N., Lagroix, F., 2015. Magnetic anisotropy reveals the depositional and postdepositional history of a loess-paleosol sequence at Nussloch (Germany). Journal of Geophysical Research-Solid Earth 120, 2859-2876.

Taylor, S.N., Lagroix, F., Rousseau, D.-D., Antoine, P., 2014. Mineral magnetic characterization of the Upper Pleniglacial Nussloch loess sequence (Germany): an insight into local environmental processes. Geophys. J. Int. 199, 1463-1480.

Ujvari, G., Stevens, T., Molnar, M., Demeny, A., Lambert, F., Varga, G., Jull, A.J.T., PallGergely, B., Buylaert, J.P., Kovacs, J., 2017. Coupled European and Greenland last glacial dust activity driven by North Atlantic climate. Proc. Natl. Acad. Sci. U.S.A. 114, E10632-E10638.

Ujvari, G., Stevens, T., Svensson, A., Klotzli, U.S., Manning, C., Nemeth, T., Kovacs, J., Sweeney, M.R., Gocke, M., Wiesenberg, G.L.B., Markovic, S.B., Zech, M., 2015. Two possible source regions for central Greenland last glacial dust. Geophys. Res. Lett. 42.

Vandenberghe, J., 2013. Grain size of fine-grained windblown sediment: a powerful proxy for process identification. Earth Sci. Rev. 121, 18-30.

Vandenberghe, J., Maddy, D., 2001. The response of river systems to climatic change. Quat. Int. 79, 1-3.

Vandenberghe, J., Markovic, S.B., Jovanovic, M., Harnbach, U., 2014. Site-specific variability of loess and palaeosols (Ruma, Vojvodina, northern Serbia). Quat. Int. 334, 86-93.

Wang, Y.J., Cheng, H., Edwards, R.L., An, Z.S., Wu, J.Y., Shen, C.C., Dorale, J.A., 2001. A high-resolution absolute-dated late Pleistocene monsoon record from Hulu Cave, China. Science 294, 2345-2348.

Wegwerth, A., Ganopolski, A., Menot, G., Kaiser, J., Dellwig, O., Bard, E., Lamy, F., Arz, H.W., 2015. Black Sea temperature response to glacial millennial-scale climate variability. Geophys. Res. Lett. 42, 8147-8154.

Woillard, G., 1978. Grande pile peat bog : a continuous pollen record for the last 140,000 years. Quat. Res. 9, 1-21.

Yang, S., Ding, Z., 2014. A 249 kyr stack of eight loess grain size records from northern China documenting millennial-scale climate variability. G-cubed 15 , $798-814$.

Zhang, X., Prange, M., Merkel, U., Schulz, M., 2014. Instability of the atlantic overturning circulation during marine isotope stage 3. Geophys. Res. Lett. 41, 4285-4293.

Zöller, L., Löscher, M., 1997. The last glacial-interglacial cycle in the loess section at Nussloch and underlying upper Tertiary loams. In: Rheinland-Pfalz, G.L. (Ed.) International Working Meeting of ISSS-Commission V and INQUA-Commission on Palaeopedolgy, Rauischholzhausen (Near Marburg, Germany), pp. 3-11. 


\section{Supplementary material}

The European loess sequences correlated in Fig. S1 have been published in the following papers with description of the pedostratigraphy, the grain size variations, $14 \mathrm{C}$ and luminescence dates.( Antoine et al., 1999; 2001; 2009a; 2009b; 2013; 2016; Fuchs et al., 2008; 2013; Hatté et al., 1998; Hatté et al., 1999; 2013; Lang et al., 2003; Moine et al., 2017; Moska et al., 2015; 2018; Rousseau et al., 2002; 2007; 2011; 2017a; 2017b; Taylor and Lagroix, 2015; Taylor et al., 2014; Tissoux et al., 2010).

Fig. S1. Correlation of the studied European sequences plotted on the same depth scale. The different colored boxes corresponds to the various Bond Cycles (BCx) discussed in the manuscript with BCA, lasting between $28,900 \mathrm{yr}$ and 23,340 yr b2k, BCB, lasting between 38,220 yr and 28,900 yr b2k, BCC, lasting between 46,860 yr and 38,220 yr b2k, and BCD, lasting between 54,220 yr and 46,860 yr b2k using Rasmussen et al (2014) chronology.

\section{References}

Antoine, P., Coutard, S., Guerin, G., Deschodt, L., Goval, E., Locht, J.L., Paris, C., 2016. Upper Pleistocene loess-palaeosol records from Northern France in the European context: Environmental background and dating of the Middle Palaeolithic. Quaternary International 411, 4-24.

Antoine, P., Rousseau, D.D., Degeai, J.P., Moine, O., Lagroix, F., kreutzer, S., Fuchs, M., Hatté, C., Gauthier, C., Svoboda, J., Lisa, L., 2013. High-resolution record of the environmental response to climatic variations during the Last Interglacial-Glacial cycle in Central Europe: The loess-palaeosol sequence of Dolní Věstonice (Czech Republic). Quaternary Science Reviews 67, 17-38.

Antoine, P., Rousseau, D.D., Fuchs, M., Hatte, C., Gauthier, C., Markovic, S.B., Jovanovic, M., Gaudenyi, T., Moine, O., Rossignol, J., 2009a. High-resolution record of the last climatic cycle in the southern Carpathian Basin (Surduk, Vojvodina, Serbia). Quaternary International 198, 19-36. 
Antoine, P., Rousseau, D.D., Lautridou, J.P., Hatté, C., 1999. Last interglacial-glacial climatic cycle in loess-paleosol successions of north-western France. Boreas 28, 551-563.

Antoine, P., Rousseau, D.D., Moine, O., Kunesch, S., Hatte, C., Lang, A., Tissoux, H., Zöller, L., 2009b. Rapid and cyclic aeolian deposition during the Last Glacial in European loess: a highresolution record from Nussloch, Germany. Quaternary Science Reviews 28, 2955-2973.

Antoine, P., Rousseau, D.D., Zöller, L., Lang, A., Munaut, A.V., Hatté, C., Fontugne, M., 2001. Highresolution record of the last interglacial-glacial cycle in the loess palaeosol sequences of Nussloch (Rhine Valley-Germany). Quaternary International 76/77, 211-229.

Fuchs, M., Kreutzer, S., Rousseau, D.-D., Antoine, P., Hatte, C., Lagroix, F., Moine, O., Gauthier, C., Svoboda, J., Lisa, L., 2013. The loess sequence of Dolni Vestonice, Czech Republic: A new OSL-based chronology of the Last Climatic Cycle. Boreas 42, 664-677.

Fuchs, M., Rousseau, D.-D., Antoine, P., Hatte, C., Gauthier, C., Markovic, S., Zoeller, L., 2008. Chronology of the last climatic cycle (upper pleistocene) of the Surduk loess sequence, Vojvodina, Serbia. Boreas 37, 66-73.

Hatté, C., Antoine, P., Fontugne, M., Rousseau, D.D., Tisnérat-Laborde, N., Zöller, L., 1999. New chronology and organic matter $\delta^{13} \mathrm{C}$ paleoclimatic significance of Nussloch loess sequence (Rhine Valley, Germany). Quaternary International 62, 85-91.

Hatté, C., Fontugne, M., Rousseau, D.D., Antoine, P., Zöller, L., Tisnérat-Laborde, N., Bentaleb, I., 1998. $\delta 13 \mathrm{C}$ variations of loess organic matter as a record of the vegetation response to climatic changes during the Weichselian. Geology 26, 583-586.

Hatté, C., Gauthier, C., Rousseau, D.D., Antoine, P., Fuchs, M., Lagroix, F., Markovic, S.B., Moine, O., Sima, A., 2013. Excursions to C4 vegetation recorded in the upper Pleistocene loess of surduk (Northern Serbia): An organic isotope geochemistry study. Climate of the Past 9, 1001-1014.

Lang, A., Hatté, C., Rousseau, D.D., Antoine, P., Fontugne, M., Zöller, L., Hambach, U., 2003. Highresolution chronologies for loess: comparing $\mathrm{AMS}^{14} \mathrm{C}$ and optical dating results. Quaternary Science Reviews 22, 953-959. 
Moine, O., Antoine, P., Hatte, C., Landais, A., Mathieu, J., Prud'homme, C., Rousseau, D.-D., 2017. The impact of Last Glacial climate variability in west-European loess revealed by radiocarbon dating of fossil earthworm granules. Proceedings of the National Academy of Sciences of the United States of America 114, 6209-6214.

Moska, P., Adamiec, G., Jary, Z., Bluszcz, A., Poreba, G., Piotrowska, N., Krawczyk, M., Skurzynski, J., 2018. Luminescence chronostratigraphy for the loess deposits in Zlota, Poland. Geochronometria 45, 44-55.

Moska, P., Jary, Z., Adamiec, G., Bluszcz, A., 2015. OSL chronostratigraphy of a loess-palaeosol sequence in Zlota using quartz and polymineral fine grains. Radiation Measurements 81, 23-31.

Rasmussen, S.O., Bigler, M., Blockley, S.P., Blunier, T., Buchardt, S.L., Clausen, H.B., Cvijanovic, I., Dahl-Jensen, D., Johnsen, S.J., Fischer, H., Gkinis, V., Guillevic, M., Hoek, W.Z., Lowe, J.J., Pedro, J.B., Popp, T., Seierstad, I.K., Steffensen, J.P., Svensson, A.M., Vallelonga, P., Vinther, B.M., Walker, M.J.C., Wheatley, J.J., Winstrup, M., 2014. A stratigraphic framework for abrupt climatic changes during the Last Glacial period based on three synchronized Greenland ice-core records: refining and extending the INTIMATE event stratigraphy. Quaternary Science Reviews 106, 14-28.

Rousseau, D.D., Antoine, P., Gerasimenko, N., Sima, A., Fuchs, M., Hatte, C., Moine, O., Zoeller, L., 2011. North Atlantic abrupt climatic events of the last glacial period recorded in Ukrainian loess deposits. Climate of the Past 7, 221-234.

Rousseau, D.D., Antoine, P., Hatté, C., Lang, A., Zöller, L., Fontugne, M., Ben Othman, D., Luck, J.M., Moine, O., Labonne, M., Bentaleb, I., Jolly, D., 2002. Abrupt millennial climatic changes from Nussloch (Germany) Upper Weichselian eolian records during the Last Glaciation. Quaternary Science Reviews 21, 1577-1582.

Rousseau, D.D., Boers, N., Sima, A., Svensson, A., Bigler, M., Lagroix, F., Taylor, S., Antoine, P., 2017a. (MIS3 \& 2) millennial oscillations in Greenland dust and Eurasian aeolian records A paleosol perspective. Quaternary Science Reviews 169, 99-113. 
Rousseau, D.D., Sima, A., Antoine, P., Hatte, C., Lang, A., Zöller, L., 2007. Link between European and North Atlantic abrupt climate changes over the last glaciation. Geophysical Research Letters 34 .

Rousseau, D.D., Svensson, A., Bigler, M., Sima, A., Steffensen, J.P., Boers, N., 2017b. Eurasian contribution to the last glacial dust cycle: how are loess sequences built? Climate of the Past 13.

Taylor, S.N., Lagroix, F., 2015. Magnetic anisotropy reveals the depositional and postdepositional history of a loess-paleosol sequence at Nussloch (Germany). Journal of Geophysical Research-Solid Earth 120, 2859-2876.

Taylor, S.N., Lagroix, F., Rousseau, D.-D., Antoine, P., 2014. Mineral magnetic characterization of the Upper Pleniglacial Nussloch loess sequence (Germany): an insight into local environmental processes. Geophysical Journal International 199, 1463-1480.

Tissoux, H., Valladas, H., Voinchet, P., Reyss, J.L., Mercier, N., Falgueres, C., Bahain, J.J., Zoeller, L., Antoine, P., 2010. OSL and ESR studies of Aeolian quartz from the Upper Pleistocene loess sequence of Nussloch (Germany). Quaternary Geochronology 5, 131-136. 


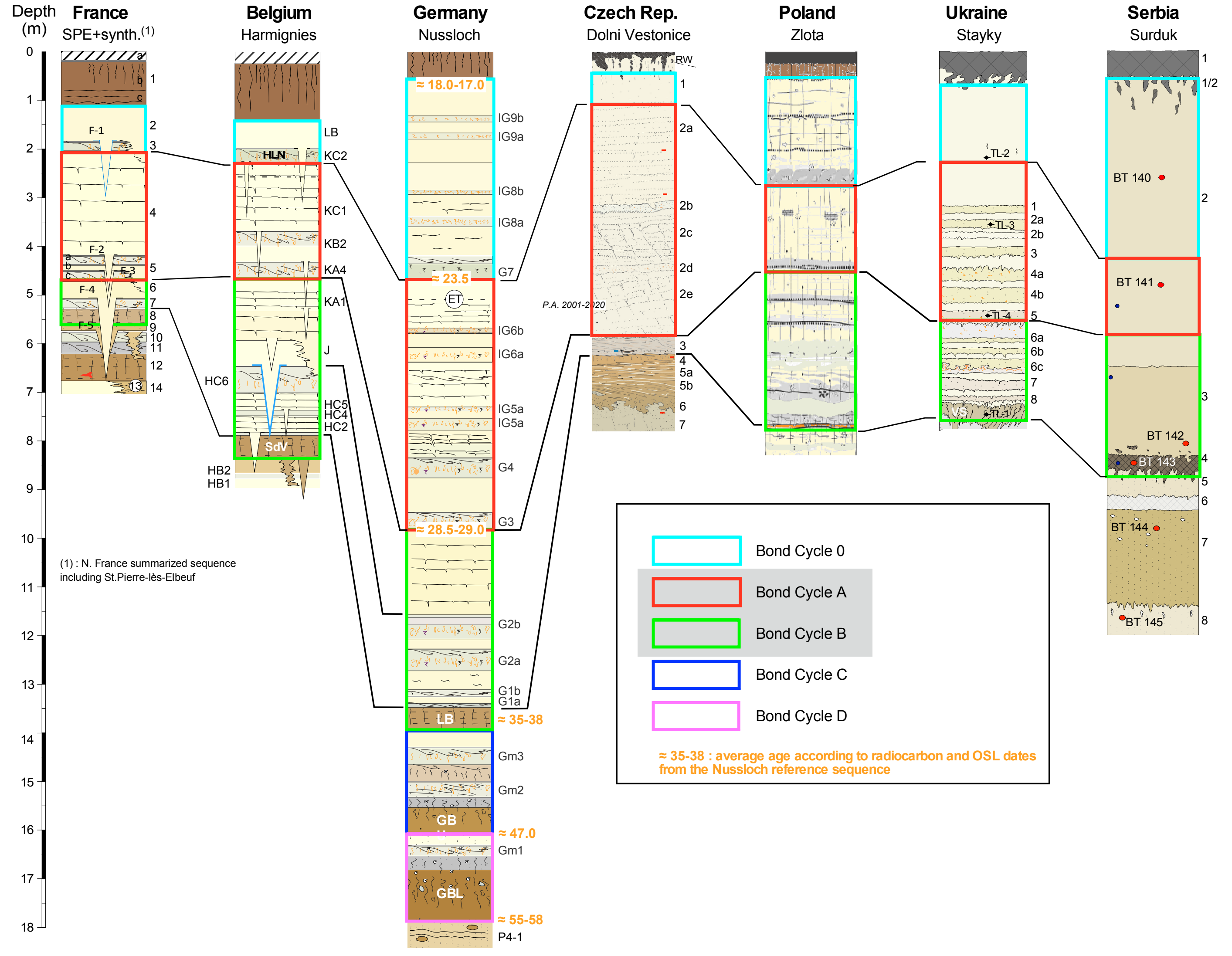

